\title{
Dual EFT bootstrap: QCD flux tubes
}

\author{
Joan Elias Miró ${ }^{a}$ and Andrea Guerrieri ${ }^{b}$ \\ ${ }^{a}$ The Abdus Salam International Centre for Theoretical Physics, \\ Strada Costiera 11, 34135, Trieste, Italy \\ ${ }^{b}$ School of Physics and Astronomy, Tel Aviv University, \\ Ramat Aviv 69978, Israel \\ E-mail: eliasmiro@ictp.it, guerrieri@mail.tau.ac.il
}

Abstract: We develop a bootstrap approach to Effective Field Theories (EFTs) based on the concept of duality in optimisation theory. As a first application, we consider the fascinating set of EFTs for confining flux tubes. The outcome of our analysis are optimal bounds on the scattering amplitude of Goldstone excitations of the flux tube, which in turn translate into bounds on the Wilson coefficients of the EFT action. Finally, we comment on how our approach compares to EFT positivity bounds.

KEywords: Effective Field Theories, Scattering Amplitudes

ArXiv EPrint: 2106.07957 


\section{Contents}

1 Introduction and motivation 1

2 Dual optimisation of Wilson coefficients 4

2.1 Primal optimisation problem 4

2.2 Dual optimisation problem 6

2.2.1 Analytic solution to Dual Problem I 9

3 Bounds on flux tubes $\quad 10$

$\begin{array}{lll}3.1 & \text { The dual problem with flavor } & 11\end{array}$

$\begin{array}{lll}3.2 & \text { Bounds } & 14\end{array}$

4 Critical amplitudes and phase-shifts $\quad 16$

5 Conclusions and outlook $\quad 18$

$\begin{array}{lr}\text { A Numerical dual problem } & 20\end{array}$

B Analytic bounds on $\gamma_{5}$ and $\gamma_{7} \quad 22$

$\begin{array}{ll}\text { C Bonus: critical manifold and log's } & 24\end{array}$

\section{Introduction and motivation}

It is widely appreciated that the paradigm of Effective Field Theory (EFT) is very much universal. However, despite the wide range of application and flexibility of EFTs, the principles of unitary evolution and causality imply very interesting bounds on the space of feasible EFTs, i.e. EFTs with a putative UV completion. A classic example is provided by the positivity bounds: while a priori Wilson coefficients can take any real value, positivity of the two-to-two forward scattering amplitude $\operatorname{Im} M>0$ implies that various Wilson coefficients are positive [1]. Many works have exploited positivity, including: the original studies in the context of the chiral Lagrangian [2-4], many interesting applications on RG-flows and the phenomenology of EFT interactions, see e.g. [5-15], as well as new developments [16-23].

Recent progress on the S-matrix bootstrap programme [24-31] has triggered a revision of the space of feasible EFTs, with applications to the EFT of: the QCD string [32], pions [33-35] and supergravity [36]. At this point a small digression is in order. Say - we are interested in the problem of finding the minimal value of a particular Wilson coefficient in an EFT action. ${ }^{1}$ We can view this task as an optimisation problem subject to the

\footnotetext{
${ }^{1}$ or the minimal value of the closely related Low Energy Constant in the scattering S-matrix.
} 
constraints dictated by unitarity and causality. There are two possible logical routes to approach the problem: a) search in the space of all physical theories, and pick the one which achieves the smallest Wilson coefficient (Primal S-matrix bootstrap); or, b) exclude all the values of the Wilson coefficient that are incompatible with either unitarity or causality, and claim a bound on the minimal Wilson coefficient (Dual S-matrix bootstrap).

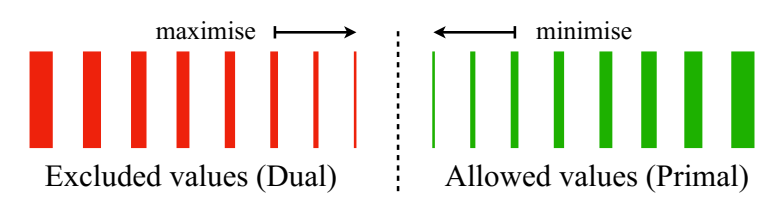

When the minimal value found from the Primal approach and the maximal of Dual approach touch each other, indicated with a dashed line above, the duality gap is closed. The concept of duality in optimisation theory has been successfully applied to bound the space of $O(N)$ models [37] and the couplings of bound states [38] in two spacetime dimensions, and quartic couplings in four spacetime dimensions $[39,40] .^{2}$ The logic of the dual S-Matrix bootstrap approach resembles that of the CFT bootstrap [45], were kinks and island are found [46-48] after excluding allowed values of the operator's scaling dimensions.

In this work we will show how to optimally bound, using a dual formulation, the allowed values of Wilson coefficients or Low Energy Constants (LECs). In order to do so we will focus our attention on the EFT of confining flux tubes $[49,50]$, see also [51-53] and references there in. This system is very fascinating per se, describing the long strings of confining three and four-dimensional theories [49, 54], and features an interesting phenomenology $[55,56]$. It also provides a simplified setting to test our ideas for bounding the space of EFTs. At low energies, the flux tube can be described by a two-dimensional action given by

$$
A=\int d^{2} \sigma \sqrt{-h}\left[\ell_{s}^{-2}+R(h)+K^{2}+\ell_{s}^{2} g_{1}\left(K_{\alpha \beta}^{\mu} K_{\mu}^{\alpha \beta}\right)^{2}+\ell_{s}^{2} g_{2} K_{\nu}^{\alpha \beta} K_{\alpha \beta}^{\mu} K_{\mu}^{\sigma \rho} K_{\sigma \rho}^{\nu}+O\left(\partial^{8}\right)\right] .
$$

The action is build out of the fields $X^{\mu}(\sigma)$, describing the embedding coordinates of the world-sheet in spacetime. In the rest of the paper we will work in units set by the string length $\ell_{s}=1$, and in the static gauge $X^{\mu}(\sigma)=\left(\sigma^{\alpha}, X^{a}\right)$, where $a=1, \ldots, D-2$. The action is invariant under the $\mathrm{SO}(D-2)$ transverse rotations, such that $X^{a}$ carries a vector (or flavour) index, and the Poincaré sub-group on the world-sheet $\operatorname{ISO}(1,1)$. The goldstone particles created by the fields $X^{a}$ are called branons.

At low energy, the leading piece in the action is the Nambu-Goto (NG) interaction $\sqrt{-h}=\sqrt{-\operatorname{det} \partial_{\alpha} X^{\mu} \partial_{\beta} X^{\nu} \eta_{\mu \nu}}$. On top of the NG interaction, and following the usual EFT logic, we include in the action any RG-irrelevant interactions that are allowed by the symmetries. Thus we include invariants build out of the intrinsic metric $h_{\alpha \beta}=\partial_{\alpha} X^{\mu} \partial_{\beta} X^{\nu} \eta_{\mu \nu}$ (like for instance the Ricci curvature scalar $R(h)$ ) and the extrinsic curvature $K_{\alpha \beta}^{\mu}=\nabla_{\alpha} \partial_{\beta} X^{\mu}$. It turns out however that $R(h)=0$ in two spacetime dimensions and that $K^{2}$ vanishes being proportional to the equations of motion. This is known as low energy universality [51, 52, 54, 57-59].

\footnotetext{
${ }^{2}$ The primal bootstrap approach to these problems was studied in [41-44], as well as [24-26, 28].
} 


\begin{tabular}{lll}
\hline & $\mathbf{D}=\mathbf{3}$ & $\mathbf{D} \geqslant \mathbf{4}$ \\
\hline Primal formulation & $\checkmark[32]$ & $\checkmark[32]$ \\
Dual formulation & $\checkmark$ section 2 & $\checkmark$ section 3 \\
Analytical solution & $\checkmark$ section 2.2 and [32] & unknown to us \\
\hline
\end{tabular}

Table 1. Optimization of low energy constants (LECs) of the flux tube EFTs.

The leading deviation from the universal NG interaction, which is sensitive to the underlying confining dynamics, arrises at order $O\left(K^{4}\right)=O\left(\partial^{6}\right)$, parametrised by $g_{1}$ and $g_{2}$ in the action (1.1). In this work we will bound the values of these non-universal interactions. In order to do so, we will use the world-sheet S-matrix, describing the scattering of the branons $X^{a}$. In particular we will need the two-to-two S-matrix, which is given by $[32,60]$

$$
\begin{aligned}
& 2 \delta_{\text {sym }}=\frac{s}{4}+\alpha_{2} s^{2}+\alpha_{3} s^{3}+O\left(s^{4}\right), \\
& 2 \delta_{\text {anti }}=\frac{s}{4}-\alpha_{2} s^{2}+\left(\alpha_{3}+2 \beta_{3}\right) s^{3}+O\left(s^{4}\right), \\
& 2 \delta_{\text {sing }}=\frac{s}{4}-(D-3) \alpha_{2} s^{2}+\left(\alpha_{3}-(D-2) \beta_{3}\right) s^{3}+O\left(s^{4}\right),
\end{aligned}
$$

where $\alpha_{2}=\frac{D-26}{384 \pi}$ is a universal one-loop contribution [51, 61], and we are using the conventional definition for the S-matrix $S_{I}(s)=\exp 2 \delta_{I}(s)$ were $I=$ sym, anti, sing. While further details are given in section 3 , note that thanks to the $\mathrm{SO}(D-2)$ symmetry $X^{a} \rightarrow$ $R_{b}^{a} X_{b}$, the two-to-two scattering can proceed in three channels (symmetric, antisymmetric and singlet), corresponding to the three irreducible representations of the incoming $\mathrm{SO}(D-$ 2) vectors $X^{a}+X^{b} \rightarrow X^{c}+X^{d}{ }^{3}$

The non-universal interactions in (1.1) are parametrised in (1.2) through $\left\{\alpha_{3}, \beta_{3}\right\} .{ }^{4}$ Our bounds on the S-matrix parameters translate into bounds on the energy levels computed in [32], which in turn can be compared against lattice Monte Carlo (MC) simulations of four-dimensional Yang-Mills. The worldsheet S-matrix approach to the QCD flux tube and its interplay with lattice MC data was pioneered in [56, 62]; see also [63] for a nice review of flux tubes from a lattice $\mathrm{MC}$ viewpoint.

In section 2 we introduce the formalism of dual EFT bootstrap. In order to do so we start discussing the flux tube in $D=3$ bulk spacetime dimensions, which has an additional pedagogic value because it is a simpler problem. In section 3 we generalize the discussion to flux tubes in general $D>3$ target spacetime dimensions and present the bounds on $\left\{\alpha_{3}, \beta_{3}\right\}$. See table 1 for a summary of what we know on the bootstrap approach to the EFT of flux tubes. A nice feature of the bootstrap approach is that it delivers the Smatrix saturating the bounds. In section 4 we discuss the phenomenology of these dual S-matrices. In 5 we conclude and discuss the interplay of positivity vs. bootstrap. Finally,

\footnotetext{
${ }^{3}$ Also recall that, after factoring out the usual delta function of total two-momenta conservation, the $S_{I}$ 's depends only on the Mandelstam variable $s=\left(p_{1}^{\mu}+p_{2}^{\mu}\right)^{2}$ because in two spacetime dimensions there is no scattering angle (i.e. $t=0$ ) and because of the Mandelstam relation $s+t+u=0$.

${ }^{4}$ In particular $\left\{\alpha_{3}, \beta_{3}\right\}=\left\{2 g_{1}+3 g_{2},-2 g_{1}-g_{2}\right\} / 8$, although the precise matching is not important for our current purposes.
} 
appendices $\mathrm{A}, \mathrm{B}$ and $\mathrm{C}$ are dedicated to give further details on the numerics, on the generalisation of $D=3$ and $D \geqslant 4$ analysis, respectively.

\section{Dual optimisation of Wilson coefficients}

In order to develop the theory of dual optimisation of Wilson coefficients, we start by analysing the scattering of a single-flavour gapless branon, a.k.a. $D=3$ flux tubes. The three processes in (1.2) reduce to a single channel $S(s)=e^{2 i \delta(s)}$, with $\delta=\delta_{\text {sing, }}$, and a single non-universal parameter is needed at $O\left(s^{3}\right), \alpha_{3}-\beta_{3} \equiv \gamma_{3}$.

The S-matrix is the boundary value of the function $S(s)$ which is analytic in the upper half plane (UHP) of the complexified Mandelstam variable $s=\left(p_{1}+p_{2}\right)^{2}$. The value of the function at specular points with respect to the imaginary axis are related by complex conjugation

$$
S\left(-z^{*}\right)=S^{*}(z),
$$

as a consequence of crossing-symmetry $S(-z)=S(z)$ and real-analyticity $S\left(z^{*}\right)=S^{*}(z)$. A nice discussion of the properties of the scattering S-matrix of massless particles in two spacetime dimensions can be found in [64]. Since $S(z)$ is the expectation value of a unitary operator it satisfies

$$
|S(s)| \leqslant 1 \quad \text { for } \quad s \in(0, \infty)
$$

i.e. for physical values of the Mandelstam variable $s$.

The spontaneously broken Poincaré invariance strongly constrains the low energy behaviour of the two-to-two phase shift $[32,65]^{5}$

$$
2 \delta(s)=\frac{s}{4}+\gamma_{3} s^{3}+\gamma_{5} s^{5}+\gamma_{7} s^{7}+O\left(s^{8}\right) .
$$

The coefficients $\gamma_{i}$ are tuneable real parameters of the low energy EFT, that should be fitted to low energy experimental data (or to MC lattice simulations data [66]), and whose precise values depend on the details of a putative UV completion. However, the $\gamma_{i}$ 's do not take arbitrary real values but instead satisfy sharp bounds that follow as a consequence of unitary (2.2), crossing and real-analyticity (2.1).

\subsection{Primal optimisation problem}

To be concrete and explain in detail the general strategy of dual optimization for Wilson coefficients, in the rest of the section we will address the specific problem of finding the minimal value of $\gamma_{3}$.

The first simple strategy to approach this problem is based on the direct numerical optimisation. In a nutshell, one introduces an ansatz for the S-matrix which encodes automatically the analytical and crossing properties (2.1), and the low energy expansion (2.3). This is for instance achieved by

$$
S_{\text {ansatz }}(\chi)=\sum_{n=0}^{n_{\max }} \alpha_{n}(\chi-1)^{n} \quad \text { with } \quad \chi(s)=\frac{s-i}{s+i},
$$

\footnotetext{
${ }^{5}$ The phase shift is real up to $O\left(s^{8}\right)$ when $2 \rightarrow 4$ particle production processes kick in.
} 
with the parameters $\left\{\alpha_{0}, \alpha_{1}, \alpha_{2}, \alpha_{3}\right\}$ fixed to match the low energy expansion $S_{\text {ansatz }}(\chi(s))$ $=\exp [i 2 \delta(s)]+O\left(s^{4}\right)(2.3)$. Next, we minimize $\gamma_{3}$ varying over the remaining $\alpha_{n \geqslant 4}$ subject to the unitary constraint (2.2). This basic logic can be generalised to higher dimensions and has been successfully used to explore the extremal values of the LECs of pion physics [33] and supergravity [36].

In the case at hand however, an analytical solution was found in [32]

$$
\gamma_{3} \geqslant-\frac{1}{768}
$$

The proof presented there is based on the Schwarz-Pick inequality. ${ }^{6}$ Consider the following function of $z$ constructed out of a physical $S$-matrix $S(z)$

$$
S^{(1)}(z \mid w) \equiv \frac{S(z)-S(w)}{1-S(z) \overline{S(w)}} / \frac{z-w}{z-\bar{w}}
$$

where $w$ is an arbitrary point in the upper half plane, and $S(z)$ maps the UHP into the unit disk. Next, note that (as a holomorphic function of $z$ in the upper half plane) this function has no singularities in the upper half plane and by unitarity is bounded by 1 for $z$ on the real line, $\left|S^{(1)}(s \mid w)\right| \leqslant 1$ for $s \in \mathbb{R}$. Then, by the maximum modulus principle, $S^{(1)}(z \mid w)$ is bounded everywhere on the upper half plane

$$
\left|S^{(1)}(z \mid w)\right| \leqslant 1 \quad \text { for } \quad z \in \mathrm{UHP} .
$$

The last equation is the content of the Schwarz-Pick theorem. Finally, inserting the low energy expansion (2.3) in the Schwarz-Pick function (2.6) and expanding for small and imaginary $z$ and $w$,

$$
S^{(1)}(i x \mid i y)=-1+\left(\frac{1}{96}+8 \gamma_{3}\right) x y+\ldots \geqslant-1,
$$

leads to (2.5). The logic flow just presented can be recursed over, i.e. one can build a $S^{(2)}$ function out of $S^{(1)}$ to bound $\gamma_{5}$, and so on. ${ }^{7}$

In the next section we will derive an alternative proof of this bound based on duality in optimization theory. ${ }^{8}$ We will work out in detail the dual formulation of the primal problem we just solved generalizing the procedure introduced in [38] for gapped theories, and highlight the various novel aspects related to gapless systems. This will clear the way for section 3 where we will be able to use the dual formulation to bootstrap max/min values of the Wilson coefficients in situations where no analytical solution is known.

\footnotetext{
${ }^{6}$ This analytic result fits in the general geometric function theory recently reviewed in [67] and generalised to other interesting physical examples.

${ }^{7}$ While further details are provided in [32], we recall that the Schwarz-Pick bounds are saturated by products of Castillejo-Dalitz-Dyson (CDD) factors (known as Blaschke products in complex analysis literature). Indeed, it is straightforward to check that the first Schwarz-Pick bound (2.5) is saturated by $S_{\text {opt }}(z)=\frac{i 8-z}{i 8+z}$. The later function is associated (i.e. equal modulo a sign) to the goldstino S-matrix that describes the flow from the Tricritical to the Critical Ising fixed points [64].

${ }^{8} \mathrm{~A}$ nice textbook is for instance [68].
} 


\subsection{Dual optimisation problem}

To derive the dual problem it is convenient to formulate the primal approach in terms of the two to two scattering amplitudes and the associated dispersion relations. The parameter $\gamma_{3}$ appears in the low energy expansion of the flux tube amplitude through (2.3), i.e.

$$
M^{\mathrm{FT}}(s)=\frac{s^{2}}{2}+\frac{i s^{3}}{16}-\left(\frac{1}{192}-2 \gamma_{3}\right) s^{4}+O\left(s^{5}\right) .
$$

The amplitude $M^{\mathrm{FT}}(s)$ is subject to unitary (2.2), and real-analyticity and crossing (2.1). ${ }^{9}$ We write the upper index in $M^{\mathrm{FT}}$ to distinguish an arbitrary amplitude from the actual flux-tube amplitude. We formulate the primal optimization problem writing all the constraints explicitly:

Primal Problem I:

Minimise $\gamma_{3}$ varying $M(s)$ constrained by

$$
\begin{aligned}
& \circ U(s) \equiv 2 \operatorname{Im} M(s)-\frac{1}{2 s}|M(s)|^{2} \geqslant 0 \text { for } s>0 \\
& \circ \operatorname{Disp}(s) \equiv \frac{1}{2} \operatorname{Re} M(s)-\frac{1}{2 \pi} \int_{0}^{\infty} \frac{s^{2}}{z^{2}} \operatorname{Im} M(z)\left(p \cdot v \cdot \frac{1}{z-s}+\frac{1}{s+z}\right) d z=0 \text { for } s>0, \\
& \circ a_{2}(0) \equiv \frac{2}{\pi} \int_{0}^{\infty} \frac{\operatorname{Im} M(z)}{z^{3}} d z=c_{2} \text { with } c_{2}=\frac{1}{2}, \\
& \circ a_{3}(0) \equiv-\frac{2}{\pi} \int_{0}^{\infty} \frac{\operatorname{Re} M(z)-c_{2} z^{2}}{z^{4}} d z=c_{3} \text { with } c_{3}=\frac{1}{16} \\
& \circ a_{4}(0) \equiv-\frac{2}{\pi} \int_{0}^{\infty} \frac{\operatorname{Im} M(z)-c_{3} z^{3}}{z^{5}} d z=c_{4} \quad \text { with } c_{4}=\frac{1}{192}-2 \gamma_{3} .
\end{aligned}
$$

Note that the constraint $(2.10 \mathrm{~b})$ is satisfied if and only if $M(s)$ is an analytic function in the UHP, which satisfies $M\left(-s^{*}\right)=M^{*}(s)$ and unitarity (2.10a). To prove the last statement we start with the following contour integral

$$
M(s)=\frac{1}{2 \pi i} \oint_{C(s)} \frac{s^{2}}{z^{2}} \frac{M(z)}{z-s} d z
$$

that encircles counter-clockwise an arbitrary point $s \in$ UHP. We introduced a double subtraction to take into account the most general behaviour at infinity compatible with unitarity (2.10a). Next we blow up the contour, use $M^{*}(z)=M\left(-z^{*}\right)$ and take $s$ real:

$$
M(s)=\frac{1}{2 \pi i} \int_{0}^{\infty} \frac{s^{2}}{z^{2}}\left(\frac{M(z)}{z-s-i 0}-\frac{M^{*}(z)}{z+s}\right) d z,
$$

\footnotetext{
${ }^{9}$ Recall that $i M(s) \equiv 2 s(S(s)-1)$, where the factor $s$ arises as a Jacobian in the relation of the identity operator of the S-matrix $\hat{S}=x \mathbb{1} S(s)$, where $\mathbb{1}=(2 \pi)^{2} s\left(\delta\left(p_{1}-p_{3}\right) \delta\left(p_{1}-p_{4}\right)+(3 \leftrightarrow 4)\right)$, and the twomomentum conservation delta in the interacting scattering amplitude $\hat{M}=(2 \pi)^{2} \delta^{(2)}\left(k_{1}^{\mu}+k_{2}^{\mu}-k_{3}^{\mu}-k_{4}^{\mu}\right) M(s)$, with $k_{i}^{\mu}=\left(\left|p_{i}\right|, p_{i}\right)$.
} 


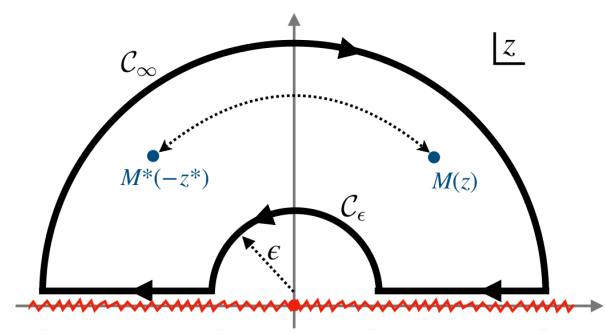

Figure 1. Contour of integration used to relate (2.13) with (2.10c)-(2.10d).

where we kept a small positive imaginary part in $s+i 0$ when needed. The double pole at $z=0$ does not pick any residue in virtue of the soft low energy behaviour of the branon amplitude (2.9). Taking the real part of the last equation, and using the Cauchy principal value (p.v.), we get (2.10b).

Regarding the low energy constraints (2.10c)-(2.10d), when analyticity and crossing (2.10b) are satisfied, we can deform the integration contours in (2.10c)-(2.10d) and write

$$
a_{n}(\epsilon)=(-1)^{n} \int_{\mathcal{C}_{\epsilon}} \frac{M(z)-\sum_{m=2}^{n-1}(-1)^{m+1}(-i z)^{m} c_{m}}{\pi(-i z)^{n+1}} d z,
$$

where $\mathcal{C}_{\epsilon}$ is a counter-clockwise semicircle contour in the UHP and centred around $z=0$, see figure 1. For $\epsilon \ll 1$, the integral in (2.13) can be evaluated using the low energy expansion in $(2.9)$

$$
a_{n}(\epsilon)=c_{n}+O(\epsilon),
$$

with the $c_{n}$ 's fixed by matching the function $M^{\mathrm{FT}}(s)=\sum_{m=2}^{9}(-1)^{m+1}(-i s)^{m} c_{m}+O\left(s^{5}\right)$ with low energy expansion (2.9). In particular, we have $a_{4}(\epsilon)=1 / 192-2 \gamma_{3}+O(\epsilon)$ when evaluating (2.13) with $M^{\mathrm{FT}}$.

Similar variables to $a_{n}(\epsilon)$ where recently used in [18], there named arcs, to study the positivity constraints of operator's Wilson coefficients along the Rernormalization Group flow. In this work, thanks to our knowledge of the low energy expansion (2.3), we have introduced subtractions in the definition of $a_{n}(\epsilon)$ such that we get (2.14).

The formulation of Primal Problem I in terms of dispersion relations pays off now because we can encode all the constraints in the following quadratic Lagrangian functional

$$
\begin{aligned}
L(\mathbf{M} ; \Lambda)= & 2 \gamma_{3}+\underbrace{\lambda_{2}\left(a_{2}(0)-c_{2}\right)+\lambda_{3}\left(a_{3}(0)-c_{3}\right)+\lambda_{4}\left(a_{4}(0)-c_{4}\right)}_{\text {low energy constants constraints }} \\
& +\underbrace{\int_{0}^{\infty}[\omega(z) \operatorname{Disp}(z)-\mu(z) U(z)] d z}_{\text {analyticity and unitarity constraints }},
\end{aligned}
$$

where $\gamma_{3}$ is our optimisation goal, and we have introduced a dual variable for each constraint in (2.10a)-(2.10e). $\mathbf{M}$ and $\Lambda$ collectively denotes all the primal and dual variables respectively

$$
\mathbf{M}=\left\{\operatorname{Re} M(z), \operatorname{Im} M(z), \gamma_{3}\right\}, \quad \Lambda=\left\{\lambda_{2}, \lambda_{3}, \lambda_{4}, \omega(z), \mu(z)\right\} .
$$


We stress that $\lambda_{2}, \lambda_{3}, \lambda_{4} \in \mathbb{R}$, and $\omega(z)$ and $\mu(z)$ are real functions defined for $z>0$, with $\mu(z)>0$ as it is related to an inequality constraint. Is is useful to think of $M(z), \omega(z)$ and $\mu(z)$ as local fields of a field-theory. While the $M(z)$ variables - one for each point in the real positive line $z \in \mathbb{R}^{+}$- are a priori arbitrary, it turns out that for $M(z)=M^{\mathrm{FT}}(z)$ in (2.9) the low energy constants constraints in (2.15) are finite.

At this point we are ready to introduce the dual functional

$$
d(\Lambda) \equiv \inf _{\mathbf{M}} L(\mathbf{M} ; \Lambda),
$$

obtained by minimising the Lagrangian w.r.t. varying $\mathbf{M}$. It turns out that $d(\Lambda)$ satisfies the following inequalities

$$
d(\Lambda) \leqslant \sup _{\Lambda} d(\Lambda)=\sup _{\Lambda} \inf _{\mathbf{M}} L(\mathbf{M} ; \Lambda) \leqslant \inf _{\mathbf{M}} \sup _{\Lambda} L(\mathbf{M} ; \Lambda)=2 \gamma_{3}^{*}
$$

where $\gamma_{3}^{*}$ is the solution to Primal Problem I. Indeed, the second inequality follows from the Min-Max theorem, switching the order of the action of sup(remum) and inf(imum). The last equality holds because $\sup _{\Lambda} L(\mathbf{M} ; \Lambda)=+\infty$ if any of the constraints is not satisfied, while $\sup _{\Lambda} L(\mathbf{M} ; \Lambda)=2 \gamma_{3}$ if $M$ is feasible, i.e. if all the constraints are satisfied. Eq. (2.18) provides the basis for formulating

\section{Dual Problem I:}

Maximize $d(\Lambda)$ varying $\Lambda=\left\{\omega(z), \mu(z), \lambda_{2}, \lambda_{3}, \lambda_{4}\right\}$, constrained by $\mu(z)>0$.

The general logic to get to formulate Dual Problem I parallels that of [38]. Next we will solve Dual Problem I and find novel aspects particular to bootstrapping EFTs. In doing so we will show that indeed the solution of Dual Problem I and Primal Problem I coincide.

In order to find $d(\Lambda)$ we will use the Euler-Lagrange equations of motion (e.o.m.) applied to (2.15). Before doing that, note that the Lagrangian (2.15) is non-local in $M(z)$ because it appears integrated over the real line in $\operatorname{Disp}(z)$, defined in $(2.10 \mathrm{~b})$. It is useful to introduce the function

$$
W\left(z^{\prime}\right)=\frac{1}{2 \pi} \int_{0}^{\infty} \omega(z) \frac{z^{2}}{z^{\prime 2}}\left(\frac{1}{z-z^{\prime}-i 0}-\frac{1}{z+z^{\prime}-i 0}\right) d z
$$

because in terms of $W$ the Lagrangian is an integral of a local density. Indeed, using $\int_{0}^{\infty} \omega(z) \operatorname{Disp}(z)=\int_{0}^{\infty} d z \operatorname{Im}(W(z) M(z)),{ }^{10}$ and the definition of the functions $a_{i}(\epsilon)$, the Lagrangian in (2.15) simplifies into

$$
L(\mathbf{M} ; \Lambda)=2 \gamma_{3}-\lambda_{2} c_{2}-\lambda_{3} c_{3}-\lambda_{4} c_{4}+\int_{0}^{\infty} d z \frac{2}{\pi} \frac{c_{2} \lambda_{3}+\lambda_{4} c_{3}}{z^{2}}+\int_{0}^{\infty} d z \operatorname{Im}(\widetilde{W}(z) M(z))-\mu(z) U(z)
$$

where we have defined

$$
\widetilde{W}(z) \equiv W(z)+\frac{2}{\pi}\left(\frac{\lambda_{2}}{z^{3}}-\frac{\lambda_{4}}{z^{5}}\right)-i \frac{2}{\pi} \frac{\lambda_{3}}{z^{4}}
$$

\footnotetext{
${ }^{10}$ It is useful to note that $\operatorname{Im} W\left(z^{\prime}\right)=\omega\left(z^{\prime}\right) / 2$ and $\operatorname{Re} W\left(z^{\prime}\right)=\frac{1}{2 \pi} \int_{0}^{\infty} \omega(z)\left(p \cdot v \cdot \frac{1}{z-z^{\prime}}+\frac{1}{z+z^{\prime}}\right) \frac{z^{2}}{z^{\prime 2}} d z$.
} 
Now we are ready to find the extrema of the functional $L(\mathbf{M} ; \Lambda) \equiv \int d z \mathcal{L}(z)$. By using the Euler-Lagrange e.o.m. $\partial_{M_{c}^{*}} \mathcal{L}=0$, and find

$$
M_{c}(z)=2 i z-\frac{i z}{\mu(z)} \widetilde{W^{*}}(z) .
$$

Moreover, the Euler-Lagrange equation $\partial_{\gamma_{3}} \mathcal{L}=0$ implies $\lambda_{4}=-1$, fixing one of the dual variables. It is easy to check that $M_{c}$ is a minimum of $L(\mathbf{M} ; \Lambda)$. Then, upon plugging the critical value of the amplitude $M_{c}$ back on the Lagrangian $L$ we are led to

$$
d(\Lambda)=\frac{1}{192}-\frac{\lambda_{2}}{2}-\frac{\lambda_{3}}{16}+\int_{0}^{\infty} d z\left[\frac{\lambda_{3}}{\pi z^{2}}-\frac{1}{8 \pi z^{2}}+2 z\left(\operatorname{Re} \widetilde{W}(z)-\mu(z)-\frac{|\widetilde{W}(z)|^{2}}{4 \mu(z)}\right)\right]
$$

where we have inserted the LECs values $\left\{c_{2}, c_{3}, c_{4}\right\}=\left\{\frac{1}{2}, \frac{1}{16}, 1 / 192-2 \gamma_{3}\right\}$.

The dual functional defined in (2.24), according to (2.18), gives a lower bound on $\gamma_{3}$ for arbitrary values of the dual variables $\Lambda=\left\{\lambda_{2}, \lambda_{3}, \omega(z), \mu(z)\right\} .{ }^{11}$ Next we will be able to find the maximal value of $d$ in (2.24) analytically. However, when considering more complicated problems in the sections below, it will be very useful to perform a numerical search of the functions that maximise expressions like (2.24).

\subsubsection{Analytic solution to Dual Problem I}

We are now in a good position to solve the Dual Problem I using the dual optimisation functional in (2.24). We start by finding the supremum of (2.24) w.r.t. varying $\mu(z)$ under the constraint $\mu(z)>0$. We get the critical function $\mu_{c}(z)=|\widetilde{W}(z)| / 2$, which substituting back to (2.24) gives

$$
\begin{aligned}
D\left(W, \lambda_{2}, \lambda_{3}\right) \equiv \sup _{\mu(z)} d(\Lambda) & =\frac{1}{192}-\frac{\lambda_{2}}{2}-\frac{\lambda_{3}}{16} \\
& +\int_{0}^{\infty} d z\left[\frac{\lambda_{3}-\frac{1}{8}}{\pi} \frac{1}{z^{2}}+2 z(\operatorname{Re} \widetilde{W}(z)-|\widetilde{W}(z)|)\right] .
\end{aligned}
$$

Next we have to maximise the dual optimisation functional $D\left(W, \lambda_{2}, \lambda_{3}\right)$ over varying $W(z)$, $\lambda_{2}$ and $\lambda_{3}$.

Here it comes an interesting aspect of the dual functional for Wilson coefficients. The integrand in (2.25) has the following low energy expansion

$$
\frac{\lambda_{3}-\frac{1}{8}}{\pi} \frac{1}{z^{2}}+z(\operatorname{Re} \widetilde{W}(z)-|\widetilde{W}(z)|)=-\frac{\left(1-4 \lambda_{3}\right)^{2}}{8 \pi z^{2}}+O(z) .
$$

The factor $-\left(1-4 \lambda_{3}\right)^{2} \leqslant 0$ is negative for $\lambda_{3} \in \mathbb{R}$. Therefore upon integrating the latest expression we find that $D\left(W, \lambda_{2}, \lambda_{3}, \lambda_{4}\right)=-\infty$, unless the residue of the second order pole vanishes. Thus, in order to maximize $D$ we must fix $\lambda_{3}=\frac{1}{4}$. All in all, we get

$$
D\left(W, \lambda_{2}, 1 / 4\right)=-\frac{1}{96}\left(1+48 \lambda_{2}\right)+\int_{0}^{\infty} d z\left[+\frac{1}{8 \pi} \frac{1}{z^{2}}+2 z(\operatorname{Re} \widetilde{W}(z)-|\widetilde{W}(z)|)\right]
$$

\footnotetext{
${ }^{11}$ The functional in (2.24) it is only convergent for particular values of the multipliers. However, it is possible to ignore this subtlety working at $\epsilon>0$, using the definitions in (2.13), and taking the limit $\epsilon \rightarrow 0$ only at the end.
} 
which is a nicely finite dual functional. We stress that the finiteness of $D\left(W, \lambda_{2}, 1 / 4\right)$, i.e. the "cancelation" of the value $-\infty$ by picking $\lambda_{3}=\frac{1}{4}$, comes out naturally as a result of maximizing $D$ over varying $\lambda_{i}$ 's.

To proceed further, we notice that the maximum is attained by picking $\operatorname{Im} W(z)=0$, which in turn using (2.20) implies $\operatorname{Re} W(z)=0 .{ }^{12}$ We are led to maximize the following functional over varying $\lambda_{2}$

$$
D\left(0, \lambda_{2}, 1 / 4\right)=-\frac{\lambda_{2}}{2}-\frac{1}{96}+\frac{1}{\pi} \int_{0}^{\infty} d z\left[+\frac{1}{8} \frac{1}{z^{2}}+\frac{4 \lambda_{2}}{z^{2}}+\frac{4}{z^{4}}-\sqrt{\left(\frac{4 \lambda_{2}}{z^{2}}+\frac{4}{z^{4}}\right)^{2}+\frac{1}{z^{6}}}\right] .
$$

It is easy to check that $\lambda_{2}=-1 / 64$ is a local maximum of $D\left(0, \lambda_{2}, 1 / 4\right)$, and it is the unique zero of $f\left(\lambda_{2}\right) \equiv \partial_{\lambda_{2}} D\left(0, \lambda_{2}, 1 / 4\right)$ because $f\left(\lambda_{2}\right)$ is absolutely monotonic. ${ }^{13}$ Therefore

$$
\sup _{\left\{W, \lambda_{2}, \lambda_{3}\right\}} D\left(W, \lambda_{2}, \lambda_{3}\right)=D(0,-1 / 64,1 / 4)=-2 \frac{1}{768},
$$

in agreement with $(2.5)$ !

We also find that the critical value of $\widetilde{W}$ is given by $\widetilde{W}_{c}(s)=\frac{2}{\pi} \frac{1}{s^{5}}-\frac{1}{32 \pi} \frac{1}{s^{3}}-i \frac{1}{2 \pi} \frac{1}{s^{4}}$. Therefore using the fat that critical scattering amplitude (2.23) is given by

$$
M_{c}(z)=2 i z\left(1-\widetilde{W_{c}^{*}}(z) / \widetilde{W_{c}}(z) \mid\right)
$$

we have $M_{c}(s)=+\frac{4 i s^{2}}{s+8 i} \cdot 14$

The formulation presented in this section can be generalised in order to bound the higher order LECs $\gamma_{5}$ and $\gamma_{7}$ in (2.3). For these more involved dual problems, we also find that the dual functional is finite when computed using the optimal $\lambda_{i}$ 's, and the extremal values of $\gamma_{5}$ and $\gamma_{7}$ coincide with the primal optimisation problem bounds of [32]. Further details are given in appendix B.

\section{Bounds on flux tubes}

In $D \geqslant 4$ there are $D-2$ transverse directions to the flux-tube. This translates into $D-2$ Goldstone bosons that transform as vectors of a $O(D-2)$ global symmetry. The scattering amplitude can be expressed in terms of three functions of the Mandelstam variable $s=$ $\left(p_{a}+p_{b}\right)^{2}$

$\left.\left.\mathbb{S}_{a b}^{d c}(s)=\sigma_{1}(s) \delta_{a b}^{c d}+\sigma_{2}(s) \delta_{a}^{c} \delta_{b}^{d}+\sigma_{3}(s) \delta_{a}^{d} \delta_{b}^{c}=\sigma_{1}(s){ }_{a}^{d} \bigcup_{b}^{c}+\sigma_{2}(s){ }_{a}^{d}\right\rangle_{b}^{c}+\sigma_{3}(s){ }_{a}^{d}\right\rangle_{b}$.

\footnotetext{
${ }^{12}$ We can find the solution by varying $\operatorname{Im} W(z)$ and $\operatorname{Re} W(z)$ as independent field variables, and then check a posteriori that the solution falls inside the constraint $(2.20)$.

${ }^{13}$ This is expected: the dual problem is always concave for minimisation (convex for maximisation) independently of the properties of the primal. This follows from the definition of the Lagrangian and from the fact that point-wise extremization is a convexity-preserving operation.

${ }^{14}$ This is similar to the Goldstino-like scattering amplitude introduced in [64] — similar bootstrap equations and bounds can be derived for the fermionic S-matrix $S(0)=-1$.
} 
These three functions describe annihilation, transmission and reflection of the vector index, as indicated by the diagrams. Crossing symmetry and real analyticity imply the following relations

$$
\sigma_{1}\left(-s^{*}\right)=\sigma_{3}(s)^{*}, \quad \sigma_{2}\left(-s^{*}\right)=\sigma_{2}(s)^{*}, \quad \sigma_{3}\left(-s^{*}\right)=\sigma_{1}(s)^{*} .
$$

Similarly to the $D=3$ case, it is therefore possible to restrict the domain of these functions to the UHP without loss of generality. The underlying $O(D-2)$ symmetry implies that the two-to-two S-matrix is diagonal when scattering two vectors in the irreps. of $O(D-2)$. Thus, the suitable linear combinations

$$
S_{\text {sing }}=(D-2) \sigma_{1}+\sigma_{2}+\sigma_{3}, \quad S_{\text {anti }}=\sigma_{2}-\sigma_{3}, \quad S_{\text {sym }}=\sigma_{2}+\sigma_{3},
$$

satisfy the diagonal unitary equation

$$
\left|S_{I}(s)\right| \leqslant 1, \quad \text { for } \quad s \in(0, \infty),
$$

where $I=\{$ sing, anti, sym $\}$, and henceforth we will use capital index $I$ to denote these channels. The amplitudes, i.e. the interacting part of the S-matrix, is defined as usual $M_{I}=2 i s\left(1-S_{I}\right)$.

For our current purposes it is useful to introduce a different basis:

$$
\begin{aligned}
& M_{1}=\frac{2 M_{\mathrm{sing}}+(D-4) M_{\mathrm{sym}}-(D-2) M_{\mathrm{anti}}}{4(D-2)}, \quad M_{2}=\frac{1}{2}\left(M_{\mathrm{sym}}+M_{\mathrm{anti}}\right), \\
& M_{3}=\frac{2 M_{\mathrm{sing}}-D M_{\mathrm{sym}}+(D-2) M_{\mathrm{anti}}}{4 i(D-2)},
\end{aligned}
$$

where crossing symmetry and real analyticity (3.2) acts on the vector $\left(M_{1}, M_{2}, M_{3}\right)$ diagonally: $M_{i}\left(-s^{*}\right)=M_{i}(s)^{*}$. In contrast to what happens in the single flavour case $(D=3)$, unitarity does not act in a simple way in the basis where crossing-symmetry is diagonal.

The low energy expansion of the flux tube (FT) amplitude defined in terms of the crossing symmetric components reads

$$
\begin{aligned}
& M_{1}^{\mathrm{FT}}=0 \times s^{2} 0 \times i s^{3} 2 \beta_{3} s^{4}+O\left(s^{5}\right), \\
& M_{2}^{\mathrm{FT}}=\frac{1}{2} s^{2}+\frac{i}{16} s^{3}-\left(\frac{1}{192}-2 \alpha_{3}-2 \beta_{3}\right) s^{4}+O\left(s^{5}\right), \\
& M_{3}^{\mathrm{FT}}=0 \times s^{2} 2 i \alpha_{2} s^{3} \frac{\alpha_{2}}{2} s^{4}+O\left(s^{5}\right) .
\end{aligned}
$$

The coefficient $\alpha_{2}=\frac{D-26}{384 \pi}$ is universal, depending only on the target space-time dimension. The Wilson coefficients $\alpha_{3}$ and $\beta_{3}$ are related to the first two non-universal corrections to the $D=4$ flux tube action.

\subsection{The dual problem with flavor}

In this section we apply the dual formalism to determine what is the allowed region in the $\left\{\alpha_{3}, \beta_{3}\right\}$ space excluding all the values of the Wilson coefficients that violate crossing, analyticity and unitarity. 
In analogy to what we have done in section 2.2 , we express each coefficient of the low energy expansion of the amplitude $M_{i}=c_{2}^{(i)} s^{2}+\ldots$ in terms of arc variables of the respective amplitudes

$$
\begin{aligned}
& a_{2}^{(i)}(\epsilon)=\frac{2}{\pi} \int_{\epsilon}^{\infty} \frac{\operatorname{Im} M_{i}(z)}{z^{3}} d z, \\
& a_{3}^{(i)}(\epsilon)=-\frac{2}{\pi} \int_{\epsilon}^{\infty} \frac{\operatorname{Re} M_{i}(z)-c_{2}^{(i)} z^{2}}{z^{4}} d z, \\
& a_{4}^{(i)}(\epsilon)=-\frac{2}{\pi} \int_{\epsilon}^{\infty} \frac{\operatorname{Im} M_{i}(z)-c_{3}^{(i)} z^{3}}{z^{5}} d z .
\end{aligned}
$$

Similarly to the previous section, the $c_{n}^{(i)}$ are read from the low energy expansion of (3.6), $M_{i}^{\mathrm{FT}}=-c_{2}^{(i)}(-i s)^{2}+c_{3}^{(i)}(-i s)^{3}-c_{4}^{(i)}(-i s)^{4}+\ldots$ The notation will look slightly more Baroque because we need to carry with us the upper flavour index. Nevertheless the logic we follow is the same as in the $D=3$.

To find the boundaries of the $\left\{\alpha_{3}, \beta_{3}\right\}$ space we choose to minimize $\alpha_{3}$ at fixed $\beta_{3} .{ }^{15}$ Thus, we formulate the following (primal) problem in terms of dispersion relations:

Primal Problem II:

Minimize $\alpha_{3}$ varying $M_{i}(s)$ constrained by

$$
\begin{aligned}
& \circ U_{I}(s) \equiv 2 \operatorname{Im} M_{I}(s)-\frac{1}{2 z}\left|M_{I}(s)\right|^{2} \geqslant 0, \quad \text { for } I \in \text { irreps, and } s>0, \\
& \circ \operatorname{Disp}_{i}(s) \equiv \frac{1}{2} \operatorname{Re} M_{i}(s)-\frac{1}{2 \pi} \int_{0}^{\infty} \frac{s^{2}}{z^{2}} \operatorname{Im} M_{i}(z)\left(p \cdot v \cdot \frac{1}{z-s}+\frac{1}{s+z}\right) d z=0 \\
& \text { for } i=1,2,3 \text { and } s>0, \\
& \circ a_{2}^{(1)}(0)=0, \quad a_{3}^{(1)}(0)=0, \quad a_{4}^{(1)}(0)=2 \beta_{3}, \\
& \circ a_{2}^{(2)}(0)=\frac{1}{2}, \quad a_{3}^{(2)}(0)=\frac{1}{16}, \quad a_{4}^{(2)}(0)=1 / 192-2 \alpha_{3}-2 \beta_{3}, \\
& \circ a_{2}^{(3)}(0)=0, \quad a_{3}^{(3)}(0)=2 \alpha_{2}, \quad a_{4}^{(3)}(0)=\alpha_{2} / 2 .
\end{aligned}
$$

We remark that (3.8a) is in the unitary basis (3.3), while (3.8b) is in the crossing-symmetric basis (3.5). In (3.8b) we took a twice subtracted dispersion relation for the three crossingsymmetric amplitudes.

The formulation of Primal Problem II is in a nice form ready for dualization. Following the same strategy explained in section 2.2 we introduce a new Lagrangian

$$
L(\mathbf{M} ; \Lambda)=\underbrace{2 \alpha_{3}}_{\text {opt. goal }}+\underbrace{\lambda_{n}^{(i)}\left(a_{n}^{(i)}(0)-c_{n}^{(i)}\right)}_{\text {LECs constraints }(3.8 \mathrm{c})-(3.8 \mathrm{e})}+\underbrace{\int_{0}^{\infty}\left[\omega_{i}(z) \operatorname{Disp}_{i}(z)+\mu_{I}(z) U_{I}(z)\right] d z}_{\text {analyticity and unitarity constraints }},
$$

\footnotetext{
${ }^{15}$ It is also possible to bound a linear combination of the two Wilson coefficients $\left(\alpha_{3}, \beta_{3}\right)=(r \cos \theta, r \sin \theta)$, with $\theta$ fixed and maximize the radius, similar to the radial optimization of $[37,38]$.
} 
with $I$ summed over $I \in\{$ sing, anti, sym $\}, i$ over $i \in\{1,2,3\}$ in the basis of (3.5), and $n \in\{2,3,4\}$. The functions $\mu_{I}(z) \geqslant 0$ are non-negative, $\omega_{i}(z)$ 's are real and we have introduced eight real dual variables $\lambda_{n}^{(i)}$, one for each of the eight low energy constraints in (3.8c)-(3.8e). The primal and dual variables are collectively denoted by

$$
\mathbf{M}=\left\{\operatorname{Re} M_{i}(z), \operatorname{Im} M_{i}(z), \alpha_{3}\right\} \quad \text { and } \quad \Lambda=\left\{\lambda_{n}^{(i)}, \omega_{i}(z), \mu_{I}(z)\right\}
$$

respectively. It is useful to introduce three analytic and anti-crossing symmetric functions $W_{i}(z)$ like $(2.20)$, such that $\int_{0}^{\infty} \omega_{i}(z) \operatorname{Disp}_{i}(z) d z=\int_{0}^{\infty} \operatorname{Im}\left(W_{i}(z) M_{i}(z)\right) d z$. It is also convenient to further simplify the Lagrangian by defining $\widetilde{W}_{i}(z) \pi / 2 \equiv W_{i}(z) \pi / 2+\lambda_{2}^{(i)} / z^{3}-$ $i \lambda_{3}^{(i)} / z^{4}-\lambda_{4}^{(i)} / z^{5}$ with $i=1,2,3$, in order to absorb in $\widetilde{W}_{i}$ the contributions coming from the $\operatorname{archs} a_{n}^{(i)}$ 's. Then, we have

$$
L(\mathbf{M} ; \Lambda)=2 \alpha_{3}-\lambda_{n}^{(i)} c_{n}^{(i)}+\int_{0}^{\infty} \frac{2}{\pi} \frac{\lambda_{3}^{(i)} c_{2}^{(i)}+\lambda_{4}^{(i)} c_{3}^{(i)}}{z^{2}}+\operatorname{Im}\left(\widetilde{W}_{i}(z) M_{i}(z)\right)+\mu_{I}(z) U_{I}(z) d z,
$$

where we left implicit the sum over $I, i$ and $n .{ }^{16}$ We introduce the dual functional

$$
d(\Lambda) \equiv \inf _{\mathbf{M}} L(\mathbf{M} ; \Lambda) .
$$

Following analogous steps to the previous section and using equation (2.18), it follows that

$$
d(\Lambda) \leqslant 2 \alpha_{3}^{*},
$$

where $\alpha_{3}^{*}$ is the solution to Primal Problem II. The last equation provides the basis for formulating

\section{Dual Problem II:}

Maximize $d(\Lambda)$ varying $\Lambda=\left\{\omega_{i}(z), \mu_{I}(z), \lambda_{a}^{(i)}\right\}$, constrained by $\mu_{I}(z)>0$.

At this point it is simple to minimize over the primal variables $M_{i}$ and $\alpha_{3}$, and derive an analytical expression for the dual functional $d(\Lambda)$. In particular, the equation of motion for $\alpha_{3}$ implies $\lambda_{4}^{(2)}=-1$. The equations of motion for $M_{I}$ are derived in a similar way to the previous section.

Given the simplicity of the dual objective, we can also maximize analytically over the multipliers $\mu_{I} \geqslant 0$. After a bit of algebra we are lead to the following dual functional

$$
\sup _{\mu_{I}(z)} d(\Lambda)=-\lambda_{n}^{(i)} c_{n}^{(i)}+\int_{0}^{\infty} \frac{2}{\pi} \frac{\lambda_{3}^{(i)} c_{2}^{(i)}+\lambda_{4}^{(i)} c_{3}^{(i)}}{z^{2}} d z+\left.\int_{0}^{\infty} \frac{z}{2} \Omega(z) d z\right|_{\lambda_{4}^{(2)}=-1},
$$

where $\Omega(z) \equiv 4 \operatorname{Re} \widetilde{W}_{2}-\left|\widetilde{W}_{1}-2 \widetilde{W}_{2}+i \widetilde{W}_{3}\right|-\frac{2}{d-2}\left|\widetilde{W}_{1}-i \widetilde{W}_{3}\right|-\frac{1}{d-2}\left|(d-4) \widetilde{W}_{1}+2(d-2) \widetilde{W}_{2}+i d \widetilde{W}_{3}\right|$.

We want to emphasise that the dual functional can be further maximized analytically by maximizing the residues of the poles of the integrand in (3.15). When the residues of the higher order poles in the expansion of $\frac{z}{2} \Omega(z)$ do not vanish, the dual functional is divergent

\footnotetext{
${ }^{16}$ E.g. $\lambda_{n}^{(i)} c_{n}^{(i)}=1 / 96+2 \alpha_{3}+2 \beta_{3}+\lambda_{2}^{(2)} / 2-11 \lambda_{3}^{(3)} /(96 \pi)+2 \beta_{3} \lambda_{4}^{(1)}$.
} 
with a definite sign, namely $d(\Lambda) \rightarrow-\infty$, hence providing a trivial (yet consistent) bound. Therefore, maximizing the residues turn out to be equivalent to set those to zero. Explicitly, for $D=4$,

$$
\frac{z}{2} \Omega(z)=\frac{4-2 \sqrt{\left(\lambda_{4}^{(3)}\right)^{2}+1}-\sqrt{\left(\lambda_{4}^{(3)}\right)^{2}+\left(\lambda_{4}^{(1)}\right)^{2}}-\sqrt{4+\left(\lambda_{4}^{(1)}\right)^{2}+\left(\lambda_{4}^{(3)}\right)^{2}+4 \lambda_{4}^{(1)}}}{\pi z^{4}}+O\left(z^{-3}\right) .
$$

In order to maximize the residue in (3.16), we find the critical values $\lambda_{4}^{(3)}=0$ and $-2 \leqslant \lambda_{4}^{(1)} \leqslant 0$. For this choice of the dual variables, the coefficient of the $z^{-4}$ and $z^{-3}$ pole of $\frac{z}{2} \Omega(z)$ vanishes. Next we look for the $1 / z^{2}$ and $1 / z$ poles of the integrand in (3.15) and cancel the corresponding residues by maximising over $\lambda_{a}^{(i)}$. Solving the system of two equations for $\lambda_{3}^{(1)}$ and $\lambda_{3}^{(2)}$ and taking the real solution we find $\left(\lambda_{3}^{(1)}, \lambda_{3}^{(2)}\right)=\left(-\lambda_{4}^{(1)} / 4,+1 / 4\right)$ All in all we find that the values

$$
\left(\lambda_{4}^{(2)}, \lambda_{4}^{(3)}, \lambda_{3}^{(1)}, \lambda_{3}^{(2)}\right)=\left(-1,0,-\lambda_{4}^{(1)} / 4,+1 / 4\right) \text { and }-2 \leqslant \lambda_{4}^{(1)} \leqslant 0,
$$

maximize the dual functional, and lead to a regular integrand in (3.15) for $z \rightarrow 0$. The value $\lambda_{4}^{(3)}=0$ trivialises the constraint $a_{4}^{(3)}=\alpha_{2} / 2$, which is fine because such constraint follows from unitarity (which we have already accounted for when integrating out $\mu_{I}(z)$ 's in (3.15)) once $a_{3}^{(i)}=c_{3}^{(i)}$ is satisfied.

Evaluating (3.15) with the critical values in (3.17) we find

$$
D\left(W_{i}, \lambda_{2}^{(i)}, \lambda_{3}^{(3)}, \lambda_{4}^{(1)}\right) \equiv-\frac{1}{96}-\frac{\lambda_{2}^{(2)}}{2}+2 \alpha_{2} \lambda_{3}^{(3)}-2 \beta_{3}\left(\lambda_{4}^{(1)}+1\right)+\int_{0}^{\infty}\left(\frac{1}{8 \pi z^{2}}+\frac{z}{2} \Omega(z)\right) d z
$$

for $D=4$. All in all we are left with the

\section{Simplified Dual Problem II:}

$$
\text { Maximize } D\left(W_{i}, \lambda_{2}^{(i)}, \lambda_{3}^{(3)}, \lambda_{4}^{(1)}\right) \text { varying }\left\{W_{i}(z), \lambda_{2}^{(i)}, \lambda_{3}^{(3)}, \lambda_{4}^{(1)}\right\} \text {. }
$$

We solve this problem in the next section.

\subsection{Bounds}

According to (3.13), evaluating $D\left(W_{i}, \lambda_{a}^{(i)}\right)$ in (3.18) with arbitrary values of the dual variables, provides a rigorous bound to the minimal value of $2 \alpha_{3}$ that can be achieved in Primal Problem II.

In order to generate bounds that are close to optimality, we consider the following class of ansatzes

$$
W_{j}^{\text {ans }}(s)=\frac{i}{s^{2}} \sum_{n=0}^{N_{*}} a_{n}^{(j)} \chi^{n}(s) \quad \text { where } \quad \chi(s)=\frac{i s_{0}-s}{i s_{0}+s},
$$

for $j=1,2,3$, and minimize $D\left(W_{i}^{\text {ans }}, \lambda_{2}^{(i)}, \lambda_{3}^{(3)}, \lambda_{4}^{(1)}\right)$ varying $\left\{a_{n}^{(j)}, \lambda_{a}^{(i)}\right\}$. The parameter $s_{0}$ is arbitrary, and we set $s_{0}=4$, in units of $\ell_{s}$. We note that as $N_{*} \rightarrow \infty,(3.20)$ characterises an arbitrary anti-crossing symmetric function $W_{j}^{\text {ans }}\left(s^{*}\right)=-W_{j}^{\text {ans }}\left(-s^{*}\right)$, analytic in the 


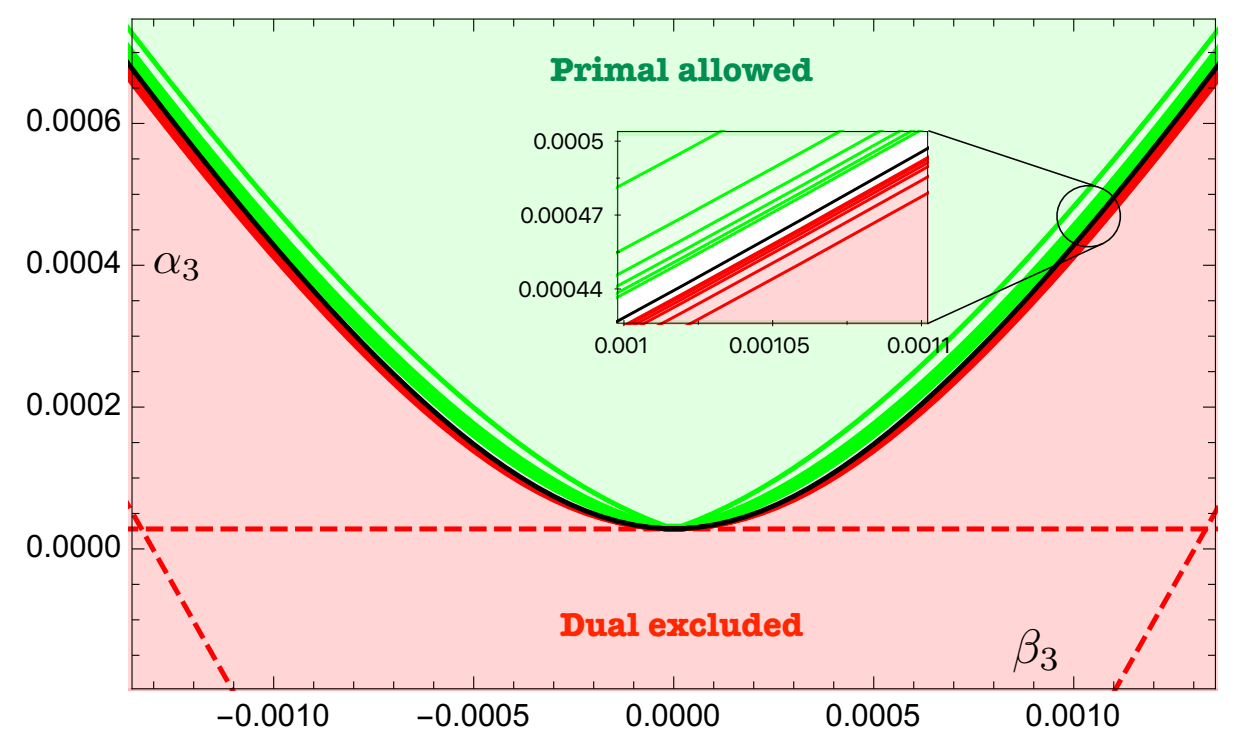

Figure 2. Primal and dual bounds on the Wilson coefficients $\left\{\alpha_{3}, \beta_{3}\right\}$. The green region is allowed by primal numerics, the red region is excluded by the dual problem. The red lines are obtained solving the dual problem at fixed $\beta_{3}$ maximizing the dual functional for $N_{*}=5,10, \ldots, 30$; the dashed red lines are the analytic bounds obtained in [32]. The green lines denote the boundary at some fixed $N_{\max }$ from $N_{\max }=20,40, \ldots, 120$; the black line is the power law extrapolation of primal numerics at $N_{*} \rightarrow \infty$. In the inset we zoom around a point of the boundary to appreciate better the convergence rate of dual numerics compared to the primal one.

UHP of $s$, and that decays as $1 / s^{2}$ as $s \rightarrow \infty$. Integrability at infinity of the dual function $D\left(W_{i}, \lambda_{a}^{(i)}\right)$ requires an ansatz decaying as $1 / s^{3}$, which we achieve imposing additional linear constraints on the $a_{n}^{(j)}$ 's. Imposing $\sum_{n}^{N_{*}}(-1)^{n} a_{n}^{(j)}=0$ guarantees $W_{j}^{\text {ans }} \sim 1 / s^{3}$ as $s \rightarrow \infty$. We allow the ansatz to have additional poles at threshold $s=0$, which are allowed from general principles and the integrability of (3.18). Intuitively, the double pole we add is 'dual' to the double zero we find in the physical amplitude $M_{i} \sim s^{2}$.

The results of the dual minimisation problem are shown in figure 2. The different red lines correspond to values of $N^{*}=5,10, \ldots, 30$, and the region below, shaded in red colour, are the values of $\left\{\alpha_{3}, \beta_{3}\right\}$ that are rigorously excluded. Needless to say, $N_{*}=30$ signifies our best exclusion bound. Convergence is so fast that on the scale of the plot the red lines are all squeezed together. We have tried variational improvement with more sophisticated ansatzes $^{17}$ which show a faster convergence. However, for the maximal $N_{*}$ that we are reporting the difference between these variational improvements is insignificant.

The green region results from primal numerics as in [32]. It is determined constructing primal solutions, namely minimising $\alpha_{3}$ at fixed $\beta_{3}$ in the space of amplitudes parametrized as in (2.4) for different $n_{\max }$ (the number of free parameters in the power series ansatz). In figure 2 the green lines correspond to values of $n_{\max }=20,40, \ldots, 120$.

Between the green and red lines there is a white space, see the zoomed in inset. That is the duality gap which we do expect to vanish once optimality is attained (or when

\footnotetext{
${ }^{17}$ Like for instance $W_{j}^{\text {ans }}(s)=\left(\frac{1}{\left(s+i z_{j}\right)^{3}}+\frac{1}{\left(s+i z_{j}\right)^{2}} \frac{R_{j}}{s}+\frac{1}{\left(s+i z_{j}\right)} \frac{R_{j+1}}{s^{2}}\right) \sum_{n=0}^{N_{*}} a_{n}^{(j)} \chi^{n}(s)$.
} 
$n_{\max } \rightarrow \infty$ and $\left.N^{*} \rightarrow \infty\right)$. We have also performed an extrapolation of the primal numerics in $n_{\max },{ }^{18}$ shown with a black curve in figure 2 . Interestingly, we find that the extrapolation of the primal falls nearly on top of the boundary of the exclusion region.

\section{Critical amplitudes and phase-shifts}

The critical amplitudes are obtained by minimising (3.11) w.r.t. $M_{i}$ and $\alpha_{3}$, and subsequently evaluating the $\mu_{I}$ dependence by maximising $d(\Lambda)$. The procedure, which is analogous to the one for $D=3$ that led to $(2.30)$, is simplified by working in the basis ${ }^{19}$

$$
\mathrm{W}_{1} \equiv \widetilde{W}_{1}-i \widetilde{W}_{3}, \quad \mathrm{~W}_{2} \equiv \widetilde{W}_{1}-2 \widetilde{W}_{2}+i \widetilde{W}_{3}, \quad \mathbb{W}_{3} \equiv(D-4) \widetilde{W}_{1}+2(D-2) \widetilde{W}_{2}+i d \widetilde{W}_{3}
$$

From the critical $M_{i}$ 's we construct the S-matrices in each irrep. and, after a bit of algebra we find:

$$
\left(S_{\text {sing }}^{D}, S_{\text {anti }}^{D}, S_{\text {sym }}^{D}\right)=\left(\frac{\mathbb{W}_{1}^{*}}{\left|\mathbb{W}_{1}\right|},-\frac{\mathbb{W}_{2}^{*}}{\left|\mathbb{W}_{2}\right|}, \frac{\mathbb{W}_{3}^{*}}{\left|\mathbb{W}_{3}\right|}\right),
$$

were the super index $D$ stands for dual. Interestingly, the dual bounds provide the dual functions that saturate $2 \rightarrow 2$ unitarity $\left|S_{I}^{D}\right|=1$. Note however that the $S_{\mathrm{I}}^{D}$ 's do not satisfy analyticity for generic values of the dual variables: this is only achieved when the duality gap closes.

In figure 3 we show the phase-shifts of the three-channels for two points in the boundary of figure 2. In each plot we show three lines: the EFT (gray), the dual (dashed) and primal (solid). The dual S-matrix phases are obtained from (4.2) while the optimal primal phaseshifts are obtained following [32]. We find that the primal and dual S-matrix phases nicely coincidence. We are showing a limited range of $s$ where the phases show the most interesting features. At larger $s$ the various phases eventually flatten.

In the left panels we plot the phase shifts for a point along the boundary with $\beta_{3}<0$, in the right panels we do the same but for $\beta_{3}>0$. Those values of $\beta_{3}$ define two phases along the boundary of the allowed region in $\left\{\alpha_{3}, \beta_{3}\right\}$ separated by the integrable point at $\beta_{3}=0$ [32]. The two phases differ by the presence of a sharp resonance respectively in the singlet (dilaton) and anti-symmetric channel (axion). In the $D=4$ case, these two phases are compatible with a symmetry of the crossing equations by exchanging singlet and anti-symmetric channels, which in turn exchanges the sign of $\beta_{3}$. Interestingly, the axion branch agrees with the expectations from approximate integrability of the QCD flux-tube: in [32] and in this work with the dual approach, we find that the axion couples to the branons with the coupling dictated by the integrable theory [69] that one would recover as the axions mass $m_{a} \rightarrow 0 .^{20}$

The plotted S-matrices allow analysing perturbative and non-perturbative physics. The perturbative physics amounts to the small momentum expansion (1.2). Comparison of the EFT amplitude with the critical amplitude informs us of the cutoff. We see that

\footnotetext{
${ }^{18}$ Done with a simple-minded power-like fit $f(x)=a+b / x^{c}$, with three free parameters $\{a, b, c\}$.

${ }^{19}$ Notice the basis (4.1) is equivalent to the unitarity basis used in [37] that makes unitarity trivial.

${ }^{20} \mathrm{It}$ is tempting to speculate that large $N$ Yang-Mills produces the integrable theory with $m_{a} \rightarrow 0$ [69]. However, lattice MC simulaitons indicate that the axion mass achieves a positive value as $N \rightarrow \infty[70]$.
} 


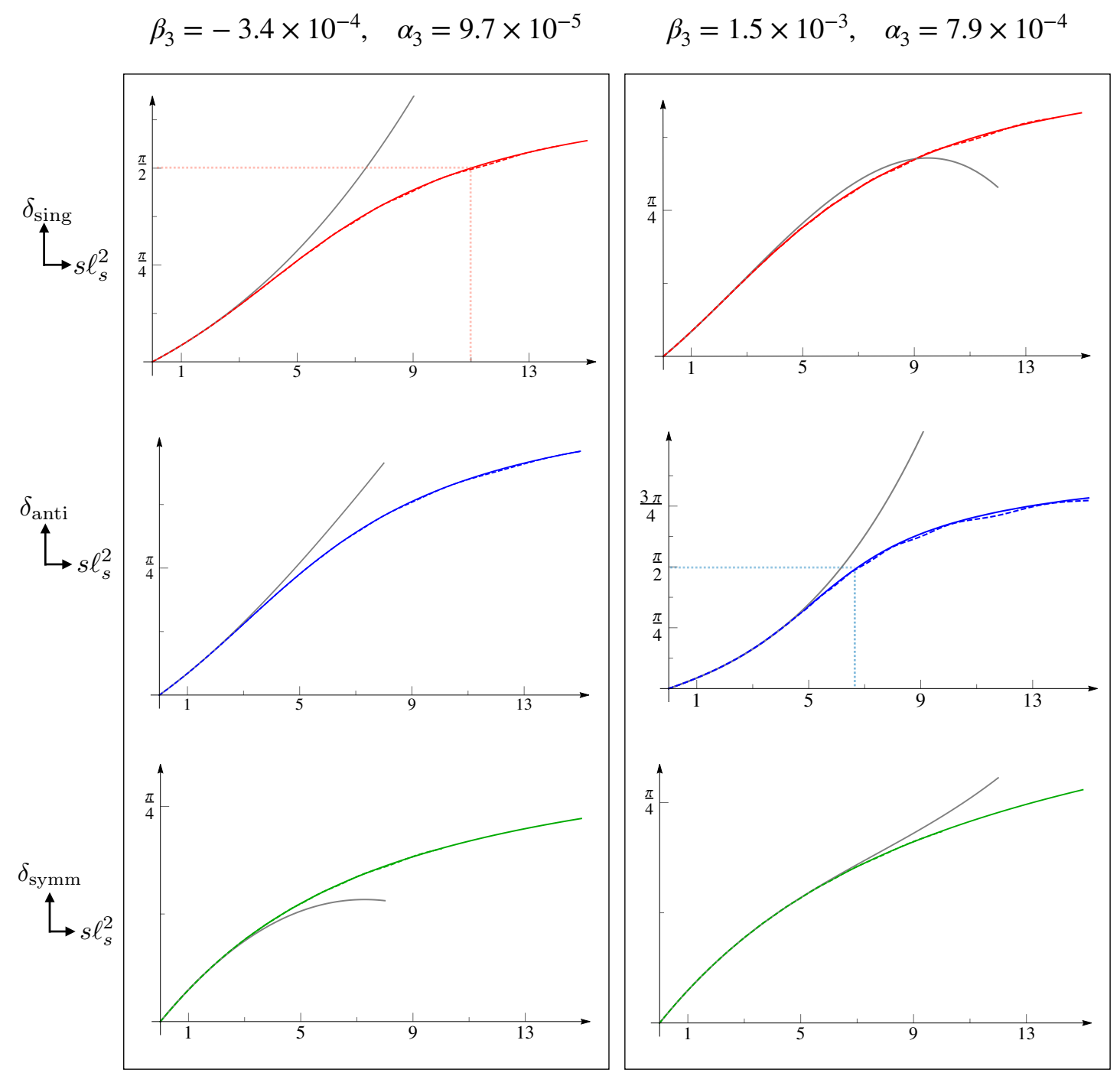

Figure 3. Phase shifts $\delta_{I}=\frac{1}{2 i} \log S_{I}$ as a function of $s \ell_{s}^{2}$ for some irrep $I$, with $I=$ ssinglet, antisymmetric, symmetric $\}$ respectively in red, blue and green. In each plot, solid line is obtained from primal numerics with $N_{\max }=120$, the dashed line is obtained from the dual with $N_{*}=30$. The gray lines are the predictions from the EFT up to two-loops. The left panel shows the phase shifts for an arbitrary $\beta_{3}<0$ : in the singlet channel there is a sharp resonance, signaled by the phase shifts passing through $\pi / 2$. The right panel shows the phase shifts for a fixed $\beta_{3}>0$ : in this case we see an axion resonance in the antisymmetric channel. Notice that for both points the EFT prediction agrees well with the non perturbative completion up to the scale set the by the lightest resonance, which, for this values of $\beta_{3}$ we chose appears dynamically around the naive cutoff scale $s^{*}=4 / \ell_{s}^{2}$.

for the actual choice of $\left\{\alpha_{3}, \beta_{3}\right\}$ the EFT validity roughly coincides with the naive EFT cutoff $s_{*}$ inferred in the IR from $s_{*} \ell_{s}^{2} / 4 \approx 1$. The actual cutoff is set dynamically by the non-perturbative phase-shifts shown in the singlet channel (left column) and antisymmetric channel (right). These two abrupt phase-shifts signal the presence of an unstable resonance. 
Finally we note that for $\beta_{3}=0$, we can find an analytic optimal solution of the dual problem. It is easy to check that

$$
\lambda_{4}^{(1)}=-1, \quad \lambda_{2}^{(3)}=\alpha_{2}, \quad \lambda_{2}^{(1)}=\lambda_{2}^{(2)}=-\frac{1}{64}+16 \alpha_{2}^{2}, \quad \lambda_{3}^{(3)}=-8 \alpha_{2}
$$

with $W_{i}=0$ is a local maximum of the dual function $D\left(W_{i}, \lambda_{2}^{(i)}, \lambda_{3}^{(3)}, \lambda_{4}^{(1)}\right)$, hence a global maximum because the dual functional is concave by definition. The analytic value of the dual functional yields the exact inequality

$$
\alpha_{3} \geqslant-\frac{1}{768}+4 \alpha_{2}^{2}
$$

The $S$-matrix saturating this bound is explicitly integrable and can be obtained plugging the dual solution (4.3) in the definition (4.2)

$$
S_{\mathrm{sing}}^{D}=S_{\mathrm{anti}}^{D}=\frac{\left(32 \alpha_{2}+i\right) s+8}{\left(32 \alpha_{2}-i\right) s+8}, \quad S_{\mathrm{sym}}^{D}=\frac{\left(-32 \alpha_{2}+i\right) s+8}{\left(-32 \alpha_{2}-i\right) s+8} .
$$

This critical S-martrix nicely coincides with the one guessed in appendix C of [32].

\section{Conclusions and outlook}

In this work we have shown how to bound the space of two-dimensional EFTs through a Smatrix bootstrap approach. For concreteness we have focused on the flux tube EFTs, which describe the long effective string sector of three and four dimensional confining theories.

As discussed in the introduction, positivity constraints on EFT Wilson coefficients has been a topic of intensive research for more than a decade. Due to the two dimensional nature of our system, we have been able to go beyond the positivity constraint by considering the full two-particle sector unitarity equation (2.10a) instead of $\operatorname{Im} M>0$. Nevertheless it is interesting to compare our methodologies with the positivity bounds widely employed in four dimensional EFTs. As a proof of concept we discuss the flux tube EFT for a single flavor (or $D=3$ flux-tube). The tree-level amplitude is

$$
M(s)=\mathrm{c}_{2} s^{2}+2 \gamma_{3} s^{4}+O\left(s^{6}\right),
$$

where $c_{2}=1 / 2$ in the normalisation of the paper. Therefore, applying the widely-known EFT positivity dispersion relation $[1],{ }^{21}$ we conclude

$$
\gamma_{3}>0 \text {, at tree-level. }
$$

In light of the perspective advocated in [18], next we improve the bound on $\gamma_{3}$ taking into account running effects, or loop corrections. For that purpose we define the arc variables

$$
\begin{aligned}
& \operatorname{arc}_{2}=\frac{2}{\pi} \int_{\epsilon}^{\infty} \frac{\operatorname{Im} M(z)}{z^{3}} d z=\mathrm{c}_{2}+O(\epsilon)>0, \\
& \operatorname{arc}_{4}=\frac{2}{\pi} \int_{\epsilon}^{\infty} \frac{\operatorname{Im} M(z)}{z^{5}} d z=\frac{\mathrm{c}_{2}^{2}}{2 \pi \epsilon}+\mathrm{c}_{4}+O(\epsilon)>0,
\end{aligned}
$$

\footnotetext{
${ }^{21}$ While it is not essential to the logic low of our analysis, we remark that in two-dimensions there has been constructions of seemingly consistent UV complete Lorentz invariant theories with the 'wrong sign' $c_{2}<0$ [71, 72], which exhibit superluminality.
} 
where the inequality signs follow from positivity $\operatorname{Im} M>0$, and recall that the loopcorrected amplitude is given by

$$
M(s)=\mathrm{c}_{2} s^{2}+\underbrace{i \mathrm{c}_{2}^{2} s^{3} / 4}_{\text {one-loop }}+(2 \gamma_{3} \underbrace{-\frac{\mathrm{c}_{2}^{3}}{24}}_{\text {two-loop }}) s^{4}+O\left(s^{5}\right) .
$$

The integrals in (5.3) are done by deforming the contour as in figure 1, and $\mathrm{c}_{4}$ is the coefficient of $s^{4}$ in (5.4). Note that due to peculiarities of two spacetime dimensions the massless cuts and naive $1 / \pi$ loop factors are absent at this order (e.g. $s^{3} \log (s)+$ crossing-symmetry $=$ $\left.s^{3} \log (s)-s^{3} \log (-s)=-i \pi s^{3}\right)$. Thus, after taking into account all loop corrections to the $O\left(s^{4}\right)$ amplitude, positivity of (5.3) implies

$$
\underbrace{2 \gamma_{3}-\frac{\mathrm{c}_{2}^{3}}{24}}_{\text {"running" } \gamma_{3}}>-\frac{\mathrm{c}_{2}^{2}}{\epsilon \pi}+O(\epsilon) .
$$

Two main points follow from (5.5): in the far IR $\epsilon \rightarrow 0$ the constraint is satisfied due to IR EFT unitarity $-\frac{c_{2}^{2}}{\epsilon \pi}<0$ (thus not sensitive to UV causality or analyticity constraints), and at intermediate energy scales the formula shows that loop corrections open a new region of parameter space allowing $\gamma_{3}$ to be negative. This is a sharp conclusion, which corrects the tree-level result (5.2).

Formula (5.5) does not allow us to precisely determine the value of the exact quantum bound on $\gamma_{3}$. Nevertheless, we do expect that such bound must exist because an arbitrarily negative $\gamma_{3}$ would produce a negative phase (2.3), which would signal non-analyticities in the UHP. ${ }^{22}$ As we have learned in this paper, such expectation is precisely addressed by the dual EFT bootstrap approach which sets the bound $\gamma_{3} \geqslant-1 / 768$. An amplitude with a $\gamma_{3}$ below such value is not feasible: it is either non-analytic in the UHP or it violates unitarity for some energy regime.

The next key step in the dual bootstrap program is to generalise the approach developed in this work to higher dimensions. Recently in $[33,36]$ it has been shown that the non perturbative bounds on pion-like and supergravity EFTs put strong constraints on the space of possible UV completions. On the other hand, for those systems the precise determination of the feasible region in the space of Wilson coefficients using the numerical S-matrix Bootstrap is a challenge. It would be very interesting to upgrade the dual EFT approach proposed in this work to higher dimensions and apply it to those and another phenomenologically relevant EFTs.

There are several questions the Dual Bootstrap might help to address in the context of two dimensional flux-tube EFTs. In [69] it was introduced the so called Axionic String Ansatz (ASA) which proposes that there are either no resonances for the $D=3$ confining flux tube, or just the axion (the resonance in the antisymmetric channel) for the $D=4$ case. Positivity bounds for the $D=3$, under the ASA hypothesis, were derived already in [32]. ${ }^{23}$

\footnotetext{
${ }^{22}$ Indeed, analyticity in the UHP implies that the total integrated phase is non-negative $\int_{-\infty}^{+\infty} d \theta \partial_{\theta} 2 \delta(\theta) \geqslant$ $0[73]$.

${ }^{23}$ See ref. [74] for a recent lattice calculation comparing the ASA for short strings against lattice MC simulations.
} 
For instance, in the $D=4$ case, we find that for $\beta_{3}<0$ the optimal S-matrix contains a sharp dilaton resonance - see figure 3 - and it would be excluded by incorporating the ASA into the Bootstrap constraints. We leave this exploration for the future.

Adding multi-particle processes to the bootstrap is a fascinating challenge both conceptually and numerically. Two-dimensional flux-tube theories are simple enough yet rich of an interesting phenomenology that would justify the effort. We believe the dual formulation might help tackling such a hard problem and perhaps single out the region where physical large- $N$ flux-tube theories might live.

We know that adding fermionic degrees of freedom and supersymmetry on the worldsheet of confining strings leads to a series of predictions for the low energy flux-tube dynamics and its S-matrix [75]. The scattering of supersymmetric gapped particles in two dimensions was studied in [76] and the bound of allowed space of couplings showed interesting geometric structures in that case. It could happen that supersymmetric world-sheet theories lies at a special point in the space of feasible Wilson coefficients. It would be interesting to study these theories with the dual bootstrap approach.

We have observed that the axion becomes lighter and that its coupling matches the integrable value as $\beta_{3}$ is increased along the boundary of figure 2 . It is tempting to imagine that, along this boundary, the axion mass $m_{a}$ decreases following a technically natural trajectory which, within perturbation theory $s \ll \ell_{s}^{-1}$, could be defined as the integrable theory in [69] softly broken by the axion mass. It will be interesting to understand how generic is this feature by checking if the resonances observed in [32], and in this work, present an analogous pattern: the mass decreases along a section of the boundary of critical Wilson coefficients and the coupling to branons matches the integrable couplings of [69]. As more couplings are turned on, it would be interesting to explore the critical manifold of the dual EFT bootstrap. Are special points (cusps, edges, ...) in this manifold of theories close to the QCD string?, and what is the spectrum of resonances along such special trajectories? It will be fascinating to analyse these questions with the dual EFT bootstrap.

\section{Acknowledgments}

We thank Sergei Dubovsky, Victor Gorbenko, Aditya Hebbar, Alexandre Homrich, Joao Penedones, Marco Serone, Amit Sever, Jacob Sonnenschein and Pedro Vieira for interesting discussions. We also thank Victor Gorbenko, Aditya Hebbar, Alexandre Homrich and Marco Serone for comments on the draft. AG is supported by The Israel Science Foundation (grant number 2289/18).

\section{A Numerical dual problem}

In this appendix we give more details about the numerical implementation of the dual problem focusing on the $D=4$ case.

As explained in section 3 , the dual problem depend on a set of real variables $\vec{\lambda}$ and three anti-crossing holomorphic functions $W_{i}(s)$ in the UHP. The space of $W_{i}(s)$ is infinite- 
dimensional, so we must truncate it choosing, for instance, a finite basis of functions. A simple choice is the Taylor series expansion

$$
W_{i}(s)=\frac{i}{s^{2}} \sum_{n=0}^{N_{i}^{\prime}} w_{n}^{(i)} \chi^{n}(s),
$$

where the function

$$
\chi(s)=-\frac{s-i z_{0}}{s+i z_{0}} .
$$

maps the upper half plane to the unit disk with centre $i z_{0} \cdot{ }^{24}$ The prime means that we eliminate one constant $w_{n}^{(i)}$ in the sum to make $W_{i} \sim 1 / s^{3}$ at large $s$. This choice is dictated by the behaviour for $z \rightarrow \infty$ of the integrand in the dual functional definition (3.18). The reader may also notice that the functional $W_{i}$ is not regular at $s=0$, but diverges as $W_{i} \sim i / s^{2}$. This divergence does not affect the convergence of the dual functional at the origin and it turns out that is needed to attain quickly the optimal bound.

To compute the integral in (3.18) numerically, we discretise the integrand on a grid of points using the Lagrange interpolation formula. We first change variable mapping the positive energy axis $s>0$ to the segment $x \in[-1,1]$ using $s(x)=z_{0} \tan \left(\frac{\pi}{4}(1+x)\right)$, then we approximate the integrand

$$
f\left(\vec{w}^{(i)}, \vec{\lambda} \mid x\right)=\frac{z_{0}}{4} \frac{\pi}{\cos ^{2}\left(\frac{\pi}{4}(1+x)\right)}\left(-\frac{1}{8 \pi^{2}} \frac{1}{s(x)^{2}}+\frac{s(x)}{2} \Omega(s(x))\right)
$$

by the interpolating polynomial of degree $N_{\text {pts }}$ passing through the $N_{\text {pts }}+1$ points $\left\{x_{k}\right\},{ }^{25}$

$$
f\left(\vec{w}^{(i)}, \vec{\lambda} \mid x\right) \approx \sum_{k=0}^{N_{\mathrm{pts}}} f\left(\vec{w}^{(i)}, \vec{\lambda} \mid x_{k}\right) \ell_{k}(x),
$$

where

$$
\ell_{k}(x)=\prod_{m \neq k} \frac{x-x_{m}}{x_{k}-x_{m}} .
$$

For the interpolation points we use the set of Chebyschev nodes $x_{k}=\cos \left(\frac{k \pi}{N_{\mathrm{pts}}+1}\right)$.

Using (A.4) we obtain an approximated expression for the dual functional

$$
D\left(\vec{w}^{(i)}, \vec{\lambda}_{i}\right) \approx-\frac{1}{96}-2 \beta_{3}-\frac{\lambda_{2}^{(2)}}{2}+2 \alpha_{2} \lambda_{3}^{(3)}-2 \beta_{3} \lambda_{4}^{(1)}+\sum_{k=0}^{N_{\mathrm{pts}}} f\left(\vec{w}^{(i)}, \vec{\lambda} \mid x_{k}\right) \int_{-1}^{1} \ell_{k}(x) d x .
$$

To search for the maximum of $D$ we use the Mathematica built in function FindMaximum.

The discretised version of the dual objective in eq. (A.6), used for the search provides a solution in terms of the dual variables $\left\{\vec{w}^{(i)}, \vec{\lambda}\right\}$. The numerical approximation does not affect the rigour of the bound since we can plug the solution found in the analytic expression (3.18) obtaining a rigorous value. We chose the number of points $N_{\text {pts }}$ large enough so that the difference between (A.6) and the analytic expression is much smaller compared to the typical values of the objective of our optimization.

\footnotetext{
${ }^{24}$ There is no obvious choice for $z_{0}$ a priori, though the rate of convergence of the numerical problem depend on its value. For our numerics we have found empirically that $z_{0} \sim 4$ gives the best convergence.

${ }^{25}$ To run the numerics we used $N_{\mathrm{pts}}=300$.
} 


\section{B Analytic bounds on $\gamma_{5}$ and $\gamma_{7}$}

In this appendix we derive the analytic shape of the $D=3$ flux-tube "Monolith" in [32], namely the 3 -dimensional allowed region in the $\gamma_{3,5,7}$ space using the dual technology developed in section 2.2.1.

We start by considering the problem of minimizing $\gamma_{5}$ for any fixed value of $\gamma_{3}$, given the low energy expansion for the S-matrix

$$
S(s)=e^{i \frac{s}{4}+i \gamma_{3} s^{3}+i \gamma_{5} s^{5}+i \gamma_{7} s^{7}}+\mathcal{O}\left(s^{8}\right) .
$$

As explained in the main text, we fix the low energy ansatz using arcs sum rules 2.13 for the amplitude $M=-2 i s(S-1)$, which explicitly yield

$$
\begin{aligned}
a_{2 k}(\epsilon) & =\frac{2(-1)^{k+1}}{\pi} \int_{\epsilon}^{\infty} \frac{\operatorname{Im} M(z)-\sum_{m=3}^{2 k-1} c_{m} \sin \frac{m \pi}{2}(-z)^{m}}{z^{2 k+1}} d z, \\
a_{2 k+1}(\epsilon) & =\frac{2(-1)^{k}}{\pi} \int_{\epsilon}^{\infty} \frac{\operatorname{Re} M(z)+\sum_{m=2}^{2 k} c_{m} \cos \frac{m \pi}{2}(-z)^{m}}{z^{2(k+1)}} d z,
\end{aligned}
$$

where the coefficients $c_{m}$ can be read off from the expansion

$$
M(s)=-\sum_{m=2}^{9}(i s)^{m} c_{m}\left(\gamma_{i}\right)+\mathcal{O}\left(s^{9}\right)
$$

using the definition in (B.1). Notice that these sum rules are valid if the amplitude we consider is analytic and crossing symmetric in the upper half plane.

So far, the derivation followed closely the one in section 2.2.1. At this point we can take a shortcut. We do not impose the dispersive constraint for any positive value of $s$, but we add just unitarity. This is not a problem, of course, since a dual bound obtained imposing a subset of constraints is still a rigorous bound. Nonetheless, it will not be generally optimal.

The Lagrangian for this problem simply reads

$$
L(M ; \Lambda)=\gamma_{5}+\sum_{n=2}^{6} \lambda_{n}\left(a_{n}(0)-c_{n}\left(\gamma_{i}\right)\right)-\int_{0}^{\infty} \mu(z) U(z) d z,
$$

with $\mu \geqslant 0$, and

$$
U(s)=2 \operatorname{Im} M(s)-\frac{|M(s)|^{2}}{2 s} \geqslant 0 .
$$

By solving the equations of motion we can solve for the $\operatorname{Re} M, \operatorname{Im} M$ and one of the $\lambda$ 's

$$
\begin{aligned}
\frac{\delta L}{\delta \operatorname{Re} M}=0 & \Longrightarrow \operatorname{Re} M=\frac{2}{\pi s^{5} \mu}\left(s^{2} \lambda_{3}-\lambda_{5}\right), \\
\frac{\delta L}{\delta \operatorname{Im} M}=0 & \Longrightarrow \operatorname{Im} M=\frac{2}{\pi s^{6} \mu}\left(s^{2} \lambda_{4}-s^{4} \lambda_{2}+\pi s^{7} \mu-\frac{1}{2}\right), \\
\frac{\partial L}{\partial \gamma_{5}} & =0 \Longrightarrow \lambda_{6}=\frac{1}{2} .
\end{aligned}
$$

Plugging this solution into the Lagrangian $L(M ; \Lambda)$ yields the dual functional $d(\Lambda)$. Before writing its explicit expression let us perform a further simplification. 
We recall that $d(\Lambda)$ is the objective of the dual problem which, in this example, provides lower bounds to the minimum value of $\gamma_{5}$ for any set of dual variables $\Lambda$. However, due to the simplicity of the Lagrangian (B.4) we can also analytically maximise $d(\Lambda)$ w.r.t. $\mu>0$, finding

$$
\mu_{c}=\frac{1}{\pi s^{3}} \sqrt{\left(\lambda_{2}-\frac{\lambda_{4}}{s^{2}}+\frac{1}{2 s^{4}}\right)^{2}+\left(\frac{\lambda_{5}}{s^{3}}-\frac{\lambda_{3}}{s}\right)^{2}} .
$$

Moreover, the function $D\left(\lambda_{i}\right) \equiv d\left(\lambda_{i}, \mu_{c}\right)$ is divergent for generic values of the dual variables. ${ }^{26}$ We find that for this problem it is sufficient to fix $\lambda_{5}=-\frac{1}{8}$ to make sure that the dual functional $D\left(\lambda_{i}\right)$ converges, yielding explicitly

$$
\begin{aligned}
D\left(\lambda_{i}\right)= & \frac{1}{2^{8} 5 !}-\frac{\gamma_{3}}{32}-\frac{\lambda_{2}}{2}-\frac{\lambda_{3}}{16}+\lambda_{4}\left(2 \gamma_{3}-\frac{1}{192}\right)+\int_{0}^{\infty} \frac{d z}{\pi}\left(\frac{1}{16 z^{4}}+\frac{\lambda_{3}+\frac{1}{8} \lambda_{4}-\frac{1}{2^{10}}}{z^{2}}\right) \\
& +\int_{0}^{\infty} d z \frac{4}{\pi z^{2}}\left(\frac{1}{2 z^{4}}-\frac{\lambda_{4}}{z^{2}}+\lambda_{2}-\sqrt{\left(\frac{1}{2 z^{4}}-\frac{\lambda_{4}}{z^{2}}+\lambda_{2}\right)^{2}+\left(\frac{1}{8 z^{3}}+\frac{\lambda_{3}}{z}\right)^{2}}\right) .
\end{aligned}
$$

By numerical inspection it turns out that the maximum of $D\left(\lambda_{i}\right)$ is attained when the integrand in eq. (B.8) vanishes. Despite the non linearity of the integrand, it is possible to set it to zero choosing $\lambda_{4}=\frac{1}{128}-8 \lambda_{3}$ and $\lambda_{2}=32 \lambda_{3}^{2}$ leaving us with a function of $\lambda_{3}$ only

$$
D\left(\lambda_{3}\right)=-16 \lambda_{3}^{2}-\left(\frac{1}{48}+16 \gamma_{3}\right)-\frac{1}{122880}-\frac{\gamma_{3}}{64}
$$

This is a concave function of $\lambda_{3}$ whose maximum is attained for $\lambda_{3}=-\frac{\gamma_{3}}{2}-\frac{1}{1536}$ producing the analytic inequality

$$
D\left(\lambda_{3}\right) \leqslant 4 \gamma_{3}^{2}-\frac{\gamma_{3}}{192}-\frac{1}{737280} \leqslant \gamma_{5}
$$

By definition, the local maximum we have found it is also global since the dual functional is a concave function of all the multipliers.

Once we find the optimal dual solution we can plug into the equation of motions (B.6) and obtain the critical $S$-matrix

$$
S=1+\frac{i}{2 s} M=\frac{8-32 \tilde{\gamma}_{3} s^{2}+i s}{8-32 \tilde{\gamma}_{3} s^{2}-i s}
$$

where $\tilde{\gamma}_{3}=\gamma_{3}+\frac{1}{768}$. For any fixed $\gamma_{3}$ this S-matrix is analytic in the upper half-plane and unitary with zeros whose location depend on the value of $\gamma_{3}$. Hence, for this problem, we find that the dual optimal solution saturates all the constraints imposed and also the analyticity constraint we have not explicitly imposed.

\footnotetext{
${ }^{26}$ It is reassuring to observe that $D\left(\lambda_{i}\right)=-\infty$ is still a lower bound, though a trivial one.
} 
The same argument can be applied to derive analytic bounds for the minimum $\gamma_{7}$ at fixed $\gamma_{3}$ and $\gamma_{5}$. Here we just report the dual optimal solution

$$
\begin{aligned}
\lambda_{2} & =-\frac{\left(64 \tilde{\gamma}_{5}-256 \tilde{\gamma}_{3}^{2}+\tilde{\gamma}_{3}\right)^{2}}{524288 \tilde{\gamma}_{3}^{2}}, \\
\lambda_{3} & =-\frac{\left(\tilde{\gamma}_{3}\left(256 \tilde{\gamma}_{3}-1\right)-64 \tilde{\gamma}_{5}\right)\left(\tilde{\gamma}_{3}+64 \tilde{\gamma}_{5}\right)}{32768 \tilde{\gamma}_{3}^{2}}, \\
\lambda_{4} & =-\frac{3}{8192}-\frac{16 \tilde{\gamma}_{5}^{2}+\tilde{\gamma}_{3} \tilde{\gamma}_{5}-2 \tilde{\gamma}_{3}^{3}}{32 \tilde{\gamma}_{3}^{2}}, \\
\lambda_{5} & =\frac{1}{256}-\frac{\tilde{\gamma}_{3}}{2}+\frac{\tilde{\gamma}_{5}}{4 \tilde{\gamma}_{3}}, \quad \lambda_{6}=-\frac{3}{128}-\frac{\tilde{\gamma}_{5}}{\tilde{\gamma}_{3}}, \\
\lambda_{7} & =\frac{1}{8}, \\
\lambda_{8} & =-\frac{1}{2} .
\end{aligned}
$$

The bound on $\gamma_{7}$ is

$$
\gamma_{7} \geqslant-\frac{1}{7340032}+\frac{\tilde{\gamma}_{3}}{4096}-\frac{\tilde{\gamma}_{3}^{2}}{16}+\frac{\tilde{\gamma}_{5}}{64}+\frac{\tilde{\gamma}_{5}^{2}}{\tilde{\gamma}_{3}}
$$

and the critical S-matrix

$$
S(s)=\frac{\left((-8+s)(-8 i+s)(8+s) \tilde{\gamma}_{3}-256 s^{3} \tilde{\gamma}_{3}^{2}+64 s^{2}(-8 i+s) \tilde{\gamma}_{5}\right)}{\left(-\left((-8+s)(8 i+s)(8+s) \tilde{\gamma}_{3}\right)+256 s^{3} \tilde{\gamma}_{3}^{2}-64 s^{2}(8 i+s) \tilde{\gamma}_{5}\right)} .
$$

\section{Bonus: critical manifold and log's}

The low energy expansion of the $D=4$ flux tube S-matrix is analytic up to $O\left(s^{5}\right)$ [75]. The first non-analytic terms are of the form $s^{5} \log s$, and are fixed by unitarity. At $O\left(s^{4}\right)$ there is a new non-universal parameter $\alpha_{4}\left(O\left(s^{5}\right)\right.$ in the M-matrix), and $O\left(s^{5}\right)$ there are two new non-universal coefficients $\left\{\alpha_{5}, \beta_{5}\right\}$ appearing in the $S$-matrix (hence $O\left(s^{6}\right)$ in the $M$-matrix). In this section we extend the dual functional introduced in the main text incorporating the parametrisation of the low energy expansion up to $O\left(s^{6}\right)$.

It turns out that (3.6) generalises into

$$
\begin{aligned}
M_{1}^{\mathrm{FT}}= & 0 \times s^{2}+0 \times i s^{3}-2 \beta_{3} s^{4}-\frac{1}{2} i \beta_{3} s^{5}+\left(\frac{1}{16} \beta_{3}-2 \beta_{5}-\frac{4}{\pi} \alpha_{2} \beta_{3} \log (-i s)\right) s^{6}+O\left(s^{7}\right) \\
M_{2}^{\mathrm{FT}}= & \frac{1}{2} s^{2}+\frac{i}{16} s^{3}-\left(\frac{1}{192}-2 \alpha_{3}-2 \beta_{3}\right) s^{4}-\frac{i}{2}\left(\frac{1}{1536}-2 \alpha_{2}^{2}-\alpha_{3}-\beta_{3}\right) s^{5} \\
& +\left(\frac{1}{61440}+2 \alpha_{5}+2 \beta_{5}-\frac{\left(2 \alpha_{2}\right)^{2}+\alpha_{3}+\beta_{3}}{16}-\frac{4}{\pi} \alpha_{2} \beta_{3} \log (-i s)\right) s^{6}+O\left(s^{7}\right) \quad(\mathrm{C} .2) \\
M_{3}^{\mathrm{FT}}= & 0 \times s^{2}+2 i \alpha_{2} s^{3}-\frac{\alpha_{2}}{2} s^{4}-i\left(\frac{\alpha_{2}}{16}-2 \alpha_{4}\right) s^{5}+\left(\frac{\alpha_{2}}{192}-2 \alpha_{2} \alpha_{3}-\frac{\alpha_{4}}{2}\right) s^{6}+O\left(s^{7}\right),
\end{aligned}
$$

where we are using the crossing-symmetric basis introduced in (3.5), and we have indicated in red and blue the appearance of the higher order non-universal parameters $\alpha_{4}$ and $\left\{\alpha_{5}, \beta_{5}\right\}$. 
Once more, we repeat the steps to formulate the dual functional. We define the Lagrangian

$$
L\left(M_{i} ; \Lambda\right)=\underbrace{\text { o.g. }}_{\text {opt. goal }}+\underbrace{\lambda_{n}^{(i)}\left(a_{n}^{(i)}(0)-c_{n}^{(i)}\right)}_{\text {LECs constraints (C.3) }}+\underbrace{\int_{0}^{\infty}\left[\omega_{i}(z) \operatorname{Disp}_{i}(z)+\mu_{I}(z) U_{I}(z)\right] d z}_{\text {analyticity and unitarity constraints }}
$$

where $\Lambda$ collectively denotes all the Lagrange multipliers $\left\{\lambda_{n}^{(i)}, \omega_{i}, \mu_{I}\right\}$; the $c_{n}^{(i)}$ are read from the low energy expansion $M_{i}^{\mathrm{FT}}=\sum_{n}^{5} s^{n} c_{n}^{(i)}+c_{6}^{(i)} s^{6}+c_{6,1}^{(i)} s^{6} \log (-i s)+O\left(s^{7}\right)$ in (C.3); and on top of (3.7) we are using

$$
\begin{aligned}
& a_{5}^{(i)}(\epsilon)=\frac{2}{\pi} \int_{\epsilon}^{\infty} \frac{\operatorname{Re} M_{i}(z)-c_{2}^{(i)} z^{2}+c_{4}^{(i)} z^{4}}{z^{6}} d z \\
& a_{6}^{(i)}(\epsilon)=\frac{2}{\pi} \int_{\epsilon}^{\infty} \frac{\operatorname{Im} M_{i}(z)-c_{3}^{(i)} z^{3}+c_{5}^{(i)} z^{5}-c_{6,1}^{(i)} z^{6} \pi / 2}{z^{7}} d z .
\end{aligned}
$$

After going through the by now familiar algebra we are led to the following dual functional

$$
\begin{aligned}
D(\Lambda) \equiv \inf _{\mu_{I}(z)} d(\Lambda)= & \text { o.g. }-\lambda_{n}^{(i)} c_{n}^{(i)}+\frac{2}{\pi} \int_{0}^{\infty} d z \frac{\lambda_{3}^{(i)} c_{2}^{(i)}+\lambda_{4}^{(i)} c_{3}^{(i)}+\lambda_{5}^{(i)} c_{4}^{(i)}+\lambda_{6}^{(i)} c_{5}^{(i)}}{z^{2}} \\
& -\frac{2}{\pi} \int_{0}^{\infty} d z \frac{\lambda_{5}^{(i)} c_{2}^{(i)}+\lambda_{6}^{(i)} c_{3}^{(i)}}{z^{4}}-\int_{0}^{\infty} d z \frac{\lambda_{6}^{(i)} c_{6,1}^{(i)}}{z}+\int_{0}^{\infty} d z \frac{z}{2} \Omega(z)
\end{aligned}
$$

where $\Omega(z) \equiv 4 \operatorname{Re} \widetilde{W}_{2}+\left|\widetilde{W}_{1}-2 \widetilde{W}_{2}+i \widetilde{W}_{3}\right|+\frac{2}{d-2}\left|\widetilde{W}_{1}-i \widetilde{W}_{3}\right|+\frac{1}{d-2}\left|(d-4) \widetilde{W}_{1}+2(d-2) \widetilde{W}_{2}+i d \widetilde{W}_{3}\right|$ and we have defined $\widetilde{W}_{i}(z) \pi / 2 \equiv W_{i}(z) \pi / 2+\lambda_{2}^{(i)} / z^{3}-i \lambda_{3}^{(i)} / z^{4}-\lambda_{4}^{(i)} / z^{5}+i \lambda_{5}^{(i)} / z^{6}+\lambda_{6}^{(i)} / z^{7}$ with $i=1,2,3$. By the same reasoning explained in the sections above, lower bounds on the minimal value of o.g. can be placed by evaluating the dual functional (C.8), and the most stringent bound are found by maximising $D(\Lambda)$ over the Lagrange multipliers.

Our next task is to remove the potential singularities $D(\Lambda) \rightarrow-\infty$ by maximising over the $\lambda_{n}^{(i)}$ 's. Again we find that dual functional is nicely finite at the maxima. In particular by fixing

$$
\left(\lambda_{6}^{(2)}, \lambda_{6}^{(3)}, \lambda_{5}^{(1)}, \lambda_{5}^{(2)}, \lambda_{5}^{(3)}\right)=\left(\lambda_{6}^{(1)}, 0,-\lambda_{6}^{(1)} / 4,-\lambda_{6}^{(1)} / 4,4 \alpha_{2} \lambda_{6}^{(1)}\right)
$$

and $\lambda_{6}^{(1)}>0$ the integrand in (C.8) is analytic around $z=0$. We have obtained bounds taking (o.g., $\left.\lambda_{6}^{(1)}, \beta_{3}\right)=\left(2 \alpha_{5}, 1,0\right)$ and scanning over $\alpha_{4}$ - but we leave for the future the detailed investigation of the critical manifold.

Open Access. This article is distributed under the terms of the Creative Commons Attribution License (CC-BY 4.0), which permits any use, distribution and reproduction in any medium, provided the original author(s) and source are credited. 


\section{References}

[1] A. Adams, N. Arkani-Hamed, S. Dubovsky, A. Nicolis and R. Rattazzi, Causality, analyticity and an IR obstruction to UV completion, JHEP 10 (2006) 014 [hep-th/0602178] [INSPIRE].

[2] T.N. Pham and T.N. Truong, Evaluation of the Derivative Quartic Terms of the Meson Chiral Lagrangian From Forward Dispersion Relation, Phys. Rev. D 31 (1985) 3027 [INSPIRE].

[3] M.R. Pennington and J. Portoles, The Chiral Lagrangian parameters, l1, l2, are determined by the rho resonance, Phys. Lett. B 344 (1995) 399 [hep-ph/9409426] [InSPIRE].

[4] B. Ananthanarayan, D. Toublan and G. Wanders, Consistency of the chiral pion pion scattering amplitudes with axiomatic constraints, Phys. Rev. D 51 (1995) 1093 [hep-ph/9410302] [INSPIRE].

[5] Z. Komargodski and A. Schwimmer, On Renormalization Group Flows in Four Dimensions, JHEP 12 (2011) 099 [arXiv:1107.3987] [INSPIRE].

[6] B. Bellazzini, Softness and amplitudes' positivity for spinning particles, JHEP 02 (2017) 034 [arXiv: 1605.06111] [INSPIRE].

[7] C. Cheung and G.N. Remmen, Positive Signs in Massive Gravity, JHEP 04 (2016) 002 [arXiv: 1601.04068] [INSPIRE].

[8] M.A. Luty, J. Polchinski and R. Rattazzi, The a-theorem and the Asymptotics of $4 D$ Quantum Field Theory, JHEP 01 (2013) 152 [arXiv:1204.5221] [INSPIRE].

[9] J. Distler, B. Grinstein, R.A. Porto and I.Z. Rothstein, Falsifying Models of New Physics via WW Scattering, Phys. Rev. Lett. 98 (2007) 041601 [hep-ph/0604255] [INSPIRE].

[10] C. Englert, G.F. Giudice, A. Greljo and M. Mccullough, The H-Parameter: An Oblique Higgs View, JHEP 09 (2019) 041 [arXiv:1903.07725] [INSPIRE].

[11] B. Bellazzini, F. Riva, J. Serra and F. Sgarlata, Beyond Positivity Bounds and the Fate of Massive Gravity, Phys. Rev. Lett. 120 (2018) 161101 [arXiv:1710. 02539] [INSPIRE].

[12] L. Alberte, C. de Rham, S. Jaitly and A.J. Tolley, QED positivity bounds, Phys. Rev. D 103 (2021) 125020 [arXiv: 2012.05798] [InSPIRE].

[13] B. Bellazzini, F. Riva, J. Serra and F. Sgarlata, Massive Higher Spins: Effective Theory and Consistency, JHEP 10 (2019) 189 [arXiv:1903.08664] [INSPIRE].

[14] J. Gu, L.-T. Wang and C. Zhang, An unambiguous test of positivity at lepton colliders, arXiv:2011.03055 [INSPIRE].

[15] C. de Rham, S. Melville, A.J. Tolley and S.-Y. Zhou, Positivity Bounds for Massive Spin-1 and Spin-2 Fields, JHEP 03 (2019) 182 [arXiv: 1804.10624] [INSPIRE].

[16] N. Arkani-Hamed, T.-C. Huang and Y.-T. Huang, The EFT-Hedron, JHEP 05 (2021) 259 [arXiv: 2012.15849] [INSPIRE].

[17] M.B. Green and C. Wen, Superstring amplitudes, unitarily, and Hankel determinants of multiple zeta values, JHEP 11 (2019) 079 [arXiv: 1908.08426] [INSPIRE].

[18] B. Bellazzini, J. Elias Miró, R. Rattazzi, M. Riembau and F. Riva, Positive moments for scattering amplitudes, Phys. Rev. D 104 (2021) 036006 [arXiv:2011.00037] [InSPIRE].

[19] A.J. Tolley, Z.-Y. Wang and S.-Y. Zhou, New positivity bounds from full crossing symmetry, JHEP 05 (2021) 255 [arXiv:2011.02400] [INSPIRE]. 
[20] S. Caron-Huot and V. Van Duong, Extremal Effective Field Theories, JHEP 05 (2021) 280 [arXiv: 2011.02957] [INSPIRE].

[21] S. Caron-Huot, D. Mazac, L. Rastelli and D. Simmons-Duffin, Sharp Boundaries for the Swampland, JHEP 07 (2021) 110 [arXiv: 2102.08951] [INSPIRE].

[22] Z. Bern, D. Kosmopoulos and A. Zhiboedov, Gravitational effective field theory islands, low-spin dominance, and the four-graviton amplitude, J. Phys. A 54 (2021) 344002 [arXiv:2103.12728] [INSPIRE].

[23] L.-Y. Chiang, Y.-t. Huang, W. Li, L. Rodina and H.-C. Weng, Into the EFThedron and UV constraints from IR consistency, arXiv:2105.02862 [INSPIRE].

[24] M.F. Paulos, J. Penedones, J. Toledo, B.C. van Rees and P. Vieira, The S-matrix bootstrap. Part I. QFT in AdS, JHEP 11 (2017) 133 [arXiv:1607.06109] [INSPIRE].

[25] M.F. Paulos, J. Penedones, J. Toledo, B.C. van Rees and P. Vieira, The S-matrix bootstrap. Part II. Two dimensional amplitudes, JHEP 11 (2017) 143 [arXiv:1607.06110] [INSPIRE].

[26] M.F. Paulos, J. Penedones, J. Toledo, B.C. van Rees and P. Vieira, The S-matrix bootstrap. Part III. Higher dimensional amplitudes, JHEP 12 (2019) 040 [arXiv:1708.06765] [INSPIRE].

[27] A.L. Guerrieri, J. Penedones and P. Vieira, Bootstrapping QCD Using Pion Scattering Amplitudes, Phys. Rev. Lett. 122 (2019) 241604 [arXiv:1810.12849] [InSPIRE].

[28] A. Homrich, J. Penedones, J. Toledo, B.C. van Rees and P. Vieira, The S-matrix Bootstrap. Part IV. Multiple Amplitudes, JHEP 11 (2019) 076 [arXiv: 1905.06905] [InSPIRE].

[29] D. Karateev, S. Kuhn and J. Penedones, Bootstrapping Massive Quantum Field Theories, JHEP 07 (2020) 035 [arXiv:1912.08940] [INSPIRE].

[30] A. Hebbar, D. Karateev and J. Penedones, Spinning S-matrix Bootstrap in $4 d$, arXiv:2011.11708 [INSPIRE].

[31] M. Correia, A. Sever and A. Zhiboedov, An Analytical Toolkit for the S-matrix Bootstrap, arXiv:2006.08221 [INSPIRE].

[32] J. Elias Miró, A.L. Guerrieri, A. Hebbar, J. Penedones and P. Vieira, Flux Tube S-matrix Bootstrap, Phys. Rev. Lett. 123 (2019) 221602 [arXiv: 1906. 08098] [INSPIRE].

[33] A.L. Guerrieri, J. Penedones and P. Vieira, S-matrix bootstrap for effective field theories: massless pions, JHEP 06 (2021) 088 [arXiv: 2011.02802] [INSPIRE].

[34] A. Bose, P. Haldar, A. Sinha, P. Sinha and S.S. Tiwari, Relative entropy in scattering and the S-matrix bootstrap, SciPost Phys. 9 (2020) 081 [arXiv:2006.12213] [INSPIRE].

[35] A. Bose, A. Sinha and S.S. Tiwari, Selection rules for the S-matrix bootstrap, SciPost Phys. 10 (2021) 122 [arXiv:2011.07944] [INSPIRE].

[36] A. Guerrieri, J. Penedones and P. Vieira, Where Is String Theory in the Space of Scattering Amplitudes?, Phys. Rev. Lett. 127 (2021) 081601 [arXiv:2102.02847] [InSPIRE].

[37] L. Córdova, Y. He, M. Kruczenski and P. Vieira, The O(N) S-matrix Monolith, JHEP 04 (2020) 142 [arXiv: 1909.06495] [InSPIRE].

[38] A.L. Guerrieri, A. Homrich and P. Vieira, Dual S-matrix bootstrap. Part I. $2 D$ theory, JHEP 11 (2020) 084 [arXiv:2008.02770] [INSPIRE]. 
[39] Y. He and M. Kruczenski, S-matrix bootstrap in $3+1$ dimensions: regularization and dual convex problem, JHEP 08 (2021) 125 [arXiv:2103.11484] [INSPIRE].

[40] A. Guerrieri and A. Sever, Rigorous bounds on the Analytic S-matrix, arXiv:2106.10257 [INSPIRE].

[41] Y. He, A. Irrgang and M. Kruczenski, A note on the S-matrix bootstrap for the $2 d O(N)$ bosonic model, JHEP 11 (2018) 093 [arXiv:1805. 02812] [INSPIRE].

[42] L. Córdova and P. Vieira, Adding flavour to the S-matrix bootstrap, JHEP 12 (2018) 063 [arXiv: 1805.11143] [INSPIRE].

[43] M.F. Paulos and Z. Zheng, Bounding scattering of charged particles in $1+1$ dimensions, JHEP 05 (2020) 145 [arXiv: 1805.11429] [INSPIRE].

[44] M. Kruczenski and H. Murali, The R-matrix bootstrap for the $2 d O(N)$ bosonic model with a boundary, JHEP 04 (2021) 097 [arXiv: 2012.15576] [INSPIRE].

[45] R. Rattazzi, V.S. Rychkov, E. Tonni and A. Vichi, Bounding scalar operator dimensions in 4D CFT, JHEP 12 (2008) 031 [arXiv:0807.0004] [INSPIRE].

[46] S. El-Showk, M.F. Paulos, D. Poland, S. Rychkov, D. Simmons-Duffin and A. Vichi, Solving the 3D Ising Model with the Conformal Bootstrap, Phys. Rev. D 86 (2012) 025022 [arXiv:1203.6064] [INSPIRE].

[47] S. El-Showk, M.F. Paulos, D. Poland, S. Rychkov, D. Simmons-Duffin and A. Vichi, Solving the 3d Ising Model with the Conformal Bootstrap II. c-Minimization and Precise Critical Exponents, J. Stat. Phys. 157 (2014) 869 [arXiv:1403.4545] [InSPIRE].

[48] F. Kos, D. Poland, D. Simmons-Duffin and A. Vichi, Precision Islands in the Ising and $O(N)$ Models, JHEP 08 (2016) 036 [arXiv: 1603.04436] [InSPIRE].

[49] M. Lüscher, Symmetry Breaking Aspects of the Roughening Transition in Gauge Theories, Nucl. Phys. B 180 (1981) 317 [InSPIRE].

[50] M. Lüscher, K. Symanzik and P. Weisz, Anomalies of the Free Loop Wave Equation in the WKB Approximation, Nucl. Phys. B 173 (1980) 365 [inSPIRE].

[51] S. Dubovsky, R. Flauger and V. Gorbenko, Effective String Theory Revisited, JHEP 09 (2012) 044 [arXiv: 1203.1054] [INSPIRE].

[52] O. Aharony and Z. Komargodski, The Effective Theory of Long Strings, JHEP 05 (2013) 118 [arXiv: 1302.6257] [INSPIRE].

[53] M. Caselle, Effective String Description of the Confining Flux Tube at Finite Temperature, Universe 7 (2021) 170 [arXiv: 2104.10486] [INSPIRE].

[54] M. Lüscher and P. Weisz, String excitation energies in $\mathrm{SU}(N)$ gauge theories beyond the free-string approximation, JHEP 07 (2004) 014 [hep-th/0406205] [INSPIRE].

[55] A. Athenodorou, B. Bringoltz and M. Teper, Closed flux tubes and their string description in $D=3+1 \mathrm{SU}(N)$ gauge theories, JHEP 02 (2011) 030 [arXiv:1007.4720] [INSPIRE].

[56] S. Dubovsky, R. Flauger and V. Gorbenko, Evidence from Lattice Data for a New Particle on the Worldsheet of the QCD Flux Tube, Phys. Rev. Lett. 111 (2013) 062006 [arXiv: 1301.2325] [INSPIRE].

[57] O. Aharony and E. Karzbrun, On the effective action of confining strings, JHEP 06 (2009) 012 [arXiv: 0903.1927] [INSPIRE]. 
[58] O. Aharony and M. Field, On the effective theory of long open strings, JHEP 01 (2011) 065 [arXiv: 1008.2636] [INSPIRE].

[59] O. Aharony and M. Dodelson, Effective String Theory and Nonlinear Lorentz Invariance, JHEP 02 (2012) 008 [arXiv:1111.5758] [INSPIRE].

[60] P. Conkey and S. Dubovsky, Four Loop Scattering in the Nambu-Goto Theory, JHEP 05 (2016) 071 [arXiv : 1603.00719] [INSPIRE].

[61] J. Polchinski and A. Strominger, Effective string theory, Phys. Rev. Lett. 67 (1991) 1681 [INSPIRE].

[62] S. Dubovsky, R. Flauger and V. Gorbenko, Flux Tube Spectra from Approximate Integrability at Low Energies, J. Exp. Theor. Phys. 120 (2015) 399 [arXiv:1404.0037] [InSPIRE].

[63] M. Teper, Large $N$ and confining flux tubes as strings - a view from the lattice, Acta Phys. Polon. B 40 (2009) 3249 [arXiv:0912.3339] [InSPIRE].

[64] A.B. Zamolodchikov, From tricritical Ising to critical Ising by thermodynamic Bethe ansatz, Nucl. Phys. B 358 (1991) 524 [InSPIRE].

[65] C. Chen, P. Conkey, S. Dubovsky and G. Hernández-Chifflet, Undressing Confining Flux Tubes with $T \bar{T}$, Phys. Rev. D 98 (2018) 114024 [arXiv:1808.01339] [INSPIRE].

[66] A. Athenodorou and M. Teper, Closed flux tubes in $D=2+1 \mathrm{SU}(N)$ gauge theories: dynamics and effective string description, JHEP 10 (2016) 093 [arXiv:1602.07634] [INSPIRE].

[67] P. Haldar, A. Sinha and A. Zahed, Quantum field theory and the Bieberbach conjecture, SciPost Phys. 11 (2021) 002 [arXiv: 2103.12108] [InSPIRE].

[68] S. Boyd and L. Vandenberghe Convex Optimization, Cambridge University Press, Cambridge U.K. (2004).

[69] S. Dubovsky and V. Gorbenko, Towards a Theory of the QCD String, JHEP 02 (2016) 022 [arXiv: 1511.01908] [INSPIRE].

[70] A. Athenodorou and M. Teper, On the mass of the world-sheet 'axion' in $\mathrm{SU}(N)$ gauge theories in $3+1$ dimensions, Phys. Lett. B 771 (2017) 408 [arXiv:1702.03717] [InSPIRE].

[71] S. Dubovsky and S. Sibiryakov, Superluminal Travel Made Possible (in two dimensions), JHEP 12 (2008) 092 [arXiv:0806.1534] [InSPIRE].

[72] P. Cooper, S. Dubovsky and A. Mohsen, Ultraviolet complete Lorentz-invariant theory with superluminal signal propagation, Phys. Rev. D 89 (2014) 084044 [arXiv:1312.2021] [INSPIRE].

[73] N. Doroud and J. Elias Miró, S-matrix bootstrap for resonances, JHEP 09 (2018) 052 [arXiv: 1804.04376] [INSPIRE].

[74] P. Conkey, S. Dubovsky and M. Teper, Glueball spins in D = 3 Yang-Mills, JHEP 10 (2019) 175 [arXiv: 1909.07430] [INSPIRE].

[75] P. Cooper, S. Dubovsky, V. Gorbenko, A. Mohsen and S. Storace, Looking for Integrability on the Worldsheet of Confining Strings, JHEP 04 (2015) 127 [arXiv:1411.0703] [INSPIRE].

[76] C. Bercini, M. Fabri, A. Homrich and P. Vieira, S-matrix bootstrap: Supersymmetry, $Z_{2}$, and $Z_{4}$ symmetry, Phys. Rev. D 101 (2020) 045022 [arXiv:1909.06453] [INSPIRE]. 